\title{
Weak approximation of stochastic differential delay equations for bounded measurable function
}

\author{
Hua Zhang
}

\begin{abstract}
In this paper we study the weak approximation problem of $E[\phi(x(T))]$ by $E[\phi(y(T))]$, where $x(T)$ is the solution of a stochastic differential delay equation and $y(T)$ is defined by the Euler scheme. For $\phi \in C_{b}^{3}$, Buckwar, Kuske, Mohammed and Shardlow ('Weak convergence of the Euler scheme for stochastic differential delay equations', LMS J. Comput. Math. 11 (2008) 60-69) have shown that the Euler scheme has weak order of convergence 1. Here we prove that the same results hold when $\phi$ is only assumed to be measurable and bounded under an additional non-degeneracy condition.
\end{abstract}

\section{Introduction}

In recent years, numerical solutions of stochastic differential equations have drawn increasing attention since they help not only to simulate the solutions of stochastic differential equations, but also have theoretical value. The Euler scheme is the simplest method among all methods of approximate numerical solutions of stochastic differential equations. There are two kinds of numerical analysis of some class of stochastic differential equations that we are interested in. The first concerns the strong convergence of the Euler scheme for some class of stochastic differential equations. The second deals with the weak approximation of some class of stochastic differential equations. More precisely, let $T>0$ be a fixed time horizon, $x(T)$ is the solution of some class of stochastic differential equations and $y(T)$ is the Euler scheme of $x(T)$ associated with a partition $\pi$, we study the following quantity

$$
E[\phi(x(T))]-E[\phi(y(T))],
$$

where $\phi$ is a suitable class of test function.

In this paper, we focus on the weak approximation of some class of differential equations due to the fact that analysis of this quantity turns out to be more important for applications, for instance, in finance, biology, and so on. Weak approximation of stochastic ordinary differential equations (SODEs) (without memory) are well developed. we refer the reader to, for example, Bally and Talay [3, 4], Kloeden and Platen [8] and Kohatsu-Higa [9] for a discussion of various aspects of this topic.

However, many physical phenomena can be modeled by stochastic dynamic systems whose evolution in time is governed by random forces as well as intrinsic dependence of the state on a finite part of its past history. Such a model may be identified as a stochastic functional differential equation (SFDE). The simplest SFDEs are stochastic delay differential equations (SDDEs) which can serve as a model of noisy physical processes whose time evolution depends on their past history. For some progress on weak approximation of the Euler scheme for SDDEs, the reader can consult [1] for a recent survey and references.

Received 9 August 2012; revised 17 April 2013.

2010 Mathematics Subject Classification 34K50, 60H07, 60H35 (primary).

This work is supported by NSF of China (No. 11171358 and 11101441), Doctor Fund of the Ministry of Education (No. 20100171110038) and Science Foundation of Guangdong Province (No. S2012040007458). 
Recent results on weak approximation of numerical methods for SDDEs are obtained by Clément, Kohatsu-Higa and Lamberton [6] and Buckwar, Kuske, Mohammed and Shardlow [1]. In [6], the authors give convergence with respect to bounded measurable test functions under sufficient non-degeneracy of the diffusion coefficient, where the drift and diffusion terms of SFDEs have the following forms

$$
b\left(\int_{-r}^{0} x(u+s) d v(s)\right) \text { and } \sigma\left(\int_{-r}^{0} x(u+s) d v(s)\right)
$$

with $v$ a finite measure on $[-r, 0]$ and $b, \sigma: \mathbb{R} \rightarrow \mathbb{R}$ sufficiently smooth real-valued functions. The result in [1] concerns the weak convergence of order 1 of the Euler scheme for fully nonlinear stochastic delay equations in $\mathbb{R}^{d}$, with multiple discrete (and continuous) delays and multidimensional Brownian noise. However, the test function $\phi$ in [1] is assumed to be $C_{b}^{3}$. In the present paper, our object is to show the existence of the expansion under a much weaker hypothesis on $\phi$. We will suppose the test function $\phi$ is measurable and bounded. The price to pay is that we should assume that the solutions of SDDEs satisfy non-degeneracy conditions. In addition to the Malliavin integration by parts formula and the tame Itô formula mentioned in [1], our approach also depends on a localization technique which was first introduced in $[\mathbf{3}, \mathbf{4}]$, and successfully applied in the proof of [6, Theorem 13].

In order to make the paper more clear, we now describe the main result of the paper and give the ideas and steps of its proof. Let $x(t ; \sigma, \eta)$ be the solution of a one-dimensional SDDE and let $y(t ; \sigma, \eta)$ be the continuous Euler approximations with a partition $\pi:=\left\{-\tau=t_{-M}<\right.$ $\left.t_{-M+1}<\ldots<t_{-1}<t_{0}=0<t_{1}<t_{2}<\ldots<t_{N-1}<t_{N}=T\right\}$ of $[-\tau, T]$, where $\sigma \in[0, T]$ is the initial instant and $\eta \in H^{1, \infty}([-\tau, 0], \mathbb{R})$ is the initial path. Here the meaning of the symbol $H^{1, \infty}([-\tau, 0], \mathbb{R})$ can be found in $\S 2$. The main result in our paper is that for a bounded measurable function $\phi: \mathbb{R} \rightarrow \mathbb{R}$, we have

$$
\left|E \phi(x(t ; \sigma, \eta))-E \phi\left(y\left(t ; \sigma, \eta^{\pi}\right)\right)\right| \leqslant C|\pi|
$$

for all $t \in[\sigma, T], \sigma \in[0, T]$ and all $\eta \in H^{1, \infty}([-\tau, 0], \mathbb{R})$, where $\eta^{\pi} \in H^{1, \infty}([-\tau, 0], \mathbb{R})$ is the piecewise-linear approximation of $\eta \in H^{1, \infty}([-\tau, 0], \mathbb{R})$. Similarly to the proof in $[\mathbf{1}]$, we only need to prove that for a bounded measurable function $\phi: \mathbb{R} \rightarrow \mathbb{R}$, the following estimate holds

$$
|E \phi(x(t ; \sigma, \eta))-E \phi(y(t ; \sigma, \eta))| \leqslant C|\pi|
$$

for all $t \in[\sigma, T], \sigma \in[0, T]$ and all $\eta \in H^{1, \infty}([-\tau, 0], \mathbb{R})$.

The general ideas and the main obstacles to overcome in the proof of the above estimate are as follows. Because $\phi$ is bounded measurable, so we can not make direct use of the method developed by Buckwar, Kuske, Mohammed and Shardlow in [1]. However, using the integration by parts formula, the test function $\phi$ can be lifted up to a test function in $C_{b}^{3}$. In order to ensure the effectiveness of the use of the integration by parts formula, we must introduce a smooth functional such that outside a set where the smooth functional vanishes, the non-degeneracy condition is satisfied. After that, using a similar procedure in [1], we can complete the proof of the estimate. More precisely, we give a brief outline of the proof of the estimate.

Step 1. Without loss of generality, we assume that $\sigma=0$ and $t=t_{n} \in \pi$ for some $0 \leqslant n \leqslant N$. Using the Markov property of $x\left(t_{n} ; 0, \eta\right)$ and $y\left(t_{n} ; 0, \eta\right)$, we have

$$
\begin{aligned}
E & {\left[\phi\left(x\left(t_{n} ; 0, \eta\right)\right)\right]-E\left[\phi\left(y\left(t_{n} ; 0, \eta\right)\right)\right] } \\
& =\sum_{i=1}^{n} E\left[\phi\left(y\left(t_{n} ; t_{i}, x_{t_{i}}\left(\cdot ; t_{i-1}, x_{t_{i-1}}(\cdot ; 0, \eta)\right)\right)\right)-\phi\left(y\left(t_{n} ; t_{i}, y_{t_{i}}\left(\cdot ; t_{i-1}, x_{t_{i-1}}(\cdot ; 0, \eta)\right)\right)\right)\right] .
\end{aligned}
$$

We consider a truncation function $\psi \in C_{b}^{\infty}([0, \infty), \mathbb{R})$ such that

$$
1_{\left[0, \frac{1}{8}\right]} \leqslant \psi \leqslant 1_{\left[0, \frac{1}{4}\right]} .
$$


We construct $r_{t_{i} t_{n}}^{\pi}$ by

$$
r_{t_{i} t_{n}}^{\pi}=\frac{\sigma_{x\left(t_{n} ; 0, \eta\right)-\frac{1}{2} y\left(t_{n} ; t_{i}, x_{t_{i}}(; ; 0, \eta)\right)-\frac{1}{2} y\left(t_{n} ; t_{i-1}, x_{t_{i-1}}(; ; 0, \eta)\right)} \vee \sigma_{y\left(t_{n} ; t_{i}, x_{t_{i}}(; ; 0, \eta)\right)-y\left(t_{n} ; t_{i-1}, x_{t_{i-1}}(; 0, \eta)\right)}}{\sigma_{x\left(t_{n} ; 0, \eta\right)}}
$$

Now we have

$$
\begin{array}{rl}
\sum_{i=1}^{n} & E\left[\phi\left(y\left(t_{n} ; t_{i}, x_{t_{i}}\left(\cdot ; t_{i-1}, x_{t_{i-1}}(\cdot ; 0, \eta)\right)\right)\right)-\phi\left(y\left(t_{n} ; t_{i}, y_{t_{i}}\left(\cdot ; t_{i-1}, x_{t_{i-1}}(\cdot ; 0, \eta)\right)\right)\right)\right] \\
= & \sum_{i=1}^{n} E\left[\left(\phi\left(y\left(t_{n} ; t_{i}, x_{t_{i}}\left(\cdot ; t_{i-1}, x_{t_{i-1}}(\cdot ; 0, \eta)\right)\right)\right)\right.\right. \\
& \left.\left.-\phi\left(y\left(t_{n} ; t_{i}, y_{t_{i}}\left(\cdot ; t_{i-1}, x_{t_{i-1}}(\cdot ; 0, \eta)\right)\right)\right)\right)\left(1-\psi\left(r_{t_{i} t_{n}}^{\pi}\right)\right)\right] \\
& +\sum_{i=1}^{n} E\left[\left(\phi\left(y\left(t_{n} ; t_{i}, x_{t_{i}}\left(\cdot ; t_{i-1}, x_{t_{i-1}}(\cdot ; 0, \eta)\right)\right)\right)\right.\right. \\
& \left.-\phi\left(y\left(t_{n} ; t_{i}, y_{t_{i}}\left(\cdot ; t_{i-1}, x_{t_{i-1}}(\cdot ; 0, \eta)\right)\right)\right) \psi\left(r_{t_{i} t_{n}}^{\pi}\right)\right] .
\end{array}
$$

Step 2. For the first part, using the definition of $\psi$, the boundedness of $\phi$ and the results of the strong convergence, we obtain

$$
\begin{aligned}
& \mid \sum_{i=1}^{n} E\left[\phi\left(y\left(t_{n} ; t_{i}, x_{t_{i}}\left(\cdot ; t_{i-1}, x_{t_{i-1}}(\cdot ; 0, \eta)\right)\right)\right)\right. \\
& \left.\quad-\phi\left(y\left(t_{n} ; t_{i}, y_{t_{i}}\left(\cdot ; t_{i-1}, x_{t_{i-1}}(\cdot ; 0, \eta)\right)\right)\right)\left(1-\psi\left(r_{t_{i} t_{n}}^{\pi}\right)\right)\right] \mid \\
& \quad \leqslant C|\pi|^{p-1} .
\end{aligned}
$$

We deal with the second part as follows. For all $0 \leqslant \lambda \leqslant 1$, we obtain

$$
\left\{\psi\left(r_{t_{i} t_{n}}^{\pi}\right) \neq 0\right\} \subset\left\{\sigma_{\lambda y\left(t_{n} ; t_{i}, x_{t_{i}}(\cdot ; 0, \eta)\right)+(1-\lambda) y\left(t_{n} ; t_{i-1}, x_{t_{i-1}}(\cdot ; 0, \eta)\right)} \geqslant \frac{1}{16} \sigma_{x\left(t_{n} ; 0, \eta\right)}\right\},
$$

and this gives that $\lambda y\left(t_{n} ; t_{i}, x_{t_{i}}(\cdot ; 0, \eta)\right)+(1-\lambda) y\left(t_{n} ; t_{i-1}, x_{t_{i-1}}(\cdot ; 0, \eta)\right)$ is nondegenerate on the set $\left\{\psi\left(r_{t_{i} t_{n}}^{\pi}\right) \neq 0\right\}$. In particular, we have $\operatorname{det}\left(\sigma_{y\left(t_{n} ; t_{i}, x_{t_{i}}(\cdot ; 0, \eta)\right)}\right)>0$ and $\operatorname{det}\left(\sigma_{y\left(t_{n} ; t_{i-1}, x_{t_{i-1}}(\cdot ; 0, \eta)\right)}\right)>0$ on the set $\left\{\psi\left(r_{t_{i} t_{n}}^{\pi}\right) \neq 0\right\}$. Then $y\left(t_{n} ; t_{i}, y_{t_{i}}\left(\cdot ; t_{i-1}, x_{t_{i-1}}(\cdot ; 0, \eta)\right)\right)$ and $y\left(t_{n} ; t_{i}, x_{t_{i}}\left(\cdot ; t_{i-1}, x_{t_{i-1}}(\cdot ; 0, \eta)\right)\right)$ have absolutely continuous law conditioned by the set $\left\{\psi\left(r_{t_{i} t_{n}}^{\pi}\right) \neq 0\right\}$. Let $\left\{\phi_{h}\right\}_{h=1}^{\infty}$ be a sequence of $C^{1}$ functions such that $\left\|\phi_{h}\right\|_{\infty} \leqslant\|\phi\|_{\infty}$ and $\left\{\phi_{h}\right\}_{h=1}^{\infty}$ converges almost every $d x$ to $\phi$ as $h$ tends to infinity. Therefore, we have

$$
\begin{aligned}
& E\left[\phi_{h}\left(y\left(t_{n} ; t_{i}, x_{t_{i}}\left(\cdot ; t_{i-1}, x_{t_{i-1}}(\cdot ; 0, \eta)\right)\right)\right) \psi\left(r_{t_{i} t_{n}}^{\pi}\right)\right] \\
& \quad \rightarrow E\left[\phi\left(y\left(t_{n} ; t_{i}, x_{t_{i}}\left(\cdot ; t_{i-1}, x_{t_{i-1}}(\cdot ; 0, \eta)\right)\right) \psi\left(r_{t_{i} t_{n}}^{\pi}\right)\right]\right.
\end{aligned}
$$

and

$$
\begin{aligned}
& E\left[\phi_{h}\left(y\left(t_{n} ; t_{i}, y_{t_{i}}\left(\cdot ; t_{i-1}, x_{t_{i-1}}(\cdot ; 0, \eta)\right)\right)\right) \psi\left(r_{t_{i} t_{n}}^{\pi}\right)\right] \\
& \quad \rightarrow E\left[\phi\left(y\left(t_{n} ; t_{i}, y_{t_{i}}\left(\cdot ; t_{i-1}, x_{t_{i-1}}(\cdot ; 0, \eta)\right)\right)\right) \psi\left(r_{t_{i} t_{n}}^{\pi}\right)\right]
\end{aligned}
$$

as $h$ tends to infinity. Thus, now it suffices to prove that

$$
\begin{aligned}
& \mid \sum_{i=1}^{n} E\left[\left(\phi_{h}\left(y\left(t_{n} ; t_{i}, x_{t_{i}}\left(\cdot ; t_{i-1}, x_{t_{i-1}}(\cdot ; 0, \eta)\right)\right)\right)\right.\right. \\
& \left.\left.\quad-\phi_{h}\left(y\left(t_{n} ; t_{i}, y_{t_{i}}\left(\cdot ; t_{i-1}, x_{t_{i-1}}(\cdot ; 0, \eta)\right)\right)\right)\right) \psi\left(r_{t_{i} t_{n}}^{\pi}\right)\right] \mid
\end{aligned}
$$


is of order $|\pi|$. Let

$$
\begin{aligned}
F_{i}= & y\left(t_{n} ; t_{i}, \lambda x_{t_{i}}\left(\cdot ; t_{i-1}, x_{t_{i-1}}(\cdot ; 0, \eta)\right)+(1-\lambda) y_{t_{i}}\left(\cdot ; t_{i-1}, x_{t_{i-1}}(\cdot ; 0, \eta)\right)\right) \\
G_{i m}= & \partial_{m} F\left(t, W(t), W\left(t_{1}\right), \ldots, W\left(t_{k}\right), s_{1}, \ldots, s_{n}, \ldots, \lambda x\left(t_{i}+\mu_{m}\right)\right. \\
& \left.+(1-\lambda) y\left(t_{i}+\mu_{m}\right), \ldots\right)\left(x_{t_{i}}\left(\cdot ; t_{i-1}, x_{t_{i-1}}(\cdot ; 0, \eta)\right)\right. \\
& -y_{t_{i}}\left(\cdot ; t_{i-1}, x_{t_{i-1}}(\cdot ; 0, \eta)\right) \psi\left(r_{t_{i} t_{n}}^{\pi}\right)
\end{aligned}
$$

Since $\lambda y\left(t_{n} ; t_{i}, x_{t_{i}}(\cdot ; 0, \eta)\right)+(1-\lambda) y\left(t_{n} ; t_{i-1}, x_{t_{i-1}}(\cdot ; 0, \eta)\right)$ is non-degenerate on the set $\left\{\psi\left(r_{t_{i} t_{n}}^{\pi}\right) \neq 0\right\}$, we use the mean value theorem, the tame character of $\nabla y(t ; 0, \eta)$ in the initial path $\eta$, and the local integration by parts formula to obtain

$$
\begin{aligned}
E & {\left[\left(\phi_{h}\left(y\left(t_{n} ; t_{i}, x_{t_{i}}\left(\cdot ; t_{i-1}, x_{t_{i-1}}(\cdot ; 0, \eta)\right)\right)\right)-\phi_{h}\left(y\left(t_{n} ; t_{i}, y_{t_{i}}\left(\cdot ; t_{i-1}, x_{t_{i-1}}(\cdot ; 0, \eta)\right)\right)\right) \psi\left(r_{t_{i} t_{n}}^{\pi}\right)\right]\right.} \\
& =\int_{0}^{1} \sum_{m=1}^{l} E\left[\nabla \phi_{h}\left(F_{i}\right) G_{i m}\right] d \lambda=\int_{0}^{1} \sum_{m=1}^{l} E\left[U\left(F_{i}\right) H_{3}\left(F_{i}, G_{i m}\right)\right] d \lambda,
\end{aligned}
$$

where $U(x)=\int_{0}^{x} \int_{0}^{y} \phi_{h}(z) d z d y$ and $H_{3}\left(F_{i}, G_{i m}\right)$ has the following representation:

$$
H_{3}\left(F_{i}, G_{i m}\right)=\sum_{k=0}^{3}\left\langle\Phi_{k}, D^{k} G_{i m}\right\rangle,
$$

with

$$
\Phi_{k} \in \mathbb{D}_{\infty}^{\infty-}\left(H^{\otimes k}\right), \quad k=0,1,2,3,
$$

which are obtained as polynomials in $\gamma_{F_{i}}, F_{i}$ and their derivatives. Thus, our problem is reformulated to prove

$$
\left|\sum_{k=0}^{3} \sum_{i=1}^{n} \sum_{m=1}^{l} \int_{0}^{1} E\left[\left\langle U\left(F_{i}\right) \Phi_{k}, D^{k} G_{i m}\right\rangle\right] d \lambda\right|
$$

is of order $|\pi|$. Now we can apply the method in [1] to obtain the desired estimate, because $U$ is in $C^{3}$ and $U^{\prime \prime}=\phi_{h}$.

Our paper is organized as follows. In $\S 2$, we recall some results of the Malliavin calculus that we will use in the following and introduce the Euler scheme for SDDEs. Our main result is given in $\S 3$ for one-dimensional SDDEs with a single delay and driven by a single Wiener process, and we put its proof in $\S 4$. In the last section, we provide a multidimensional version of our main result.

\section{Preliminary}

Now let us recall and fix some notation and notions. Let $(B, H, \mu)$ be an abstract Wiener space. We refer to [14] (see also [7] and [11]) for the background in the Malliavin calculus. The Malliavin calculus has been developed as a differential-integral calculus or a Schwartz distribution theory on $B$. The main ingredients are notions of differential operators like the gradient operator (or Shigekawa's $H$-derivative) $D$, its dual divergence operator (or the Skorohod operator) $\delta$, the Ornstein-Uhlenbeck operator $L=-\delta D$ and notions of Sobolev space $\mathbb{D}_{\alpha}^{p}, 1<p<\infty, \alpha \in \mathbb{R}$, of real Wiener and generalized Wiener functionals. Roughly,

$$
\mathbb{D}_{\alpha}^{p}=(1-L)^{-\alpha / 2}\left(\mathbb{L}^{p}\right)
$$

with the norm

$$
\|F\|_{\alpha, p}=\left\|(1-L)^{\alpha / 2} F\right\|_{\mathbb{L}^{p}},
$$

where $\mathbb{L}^{p}$ is the usual $\mathbb{L}^{p}$-space. In particular,

$$
\mathbb{D}_{0}^{p}=\mathbb{L}^{p}, \quad \mathbb{D}_{\alpha^{\prime}}^{p^{\prime}} \subseteq \mathbb{D}_{\alpha}^{p} \quad \text { if } p<p^{\prime} \text { and } \alpha<\alpha^{\prime} .
$$


So, roughly, $F \in \mathbb{D}_{\alpha}^{p}$ if and only if the derivatives of $F$ up to the $\alpha$ th order belong to $\mathbb{L}^{p}$ and, therefore, $p$ and $\alpha$ are called the integrability index and the differentiability index of the Sobolev space, respectively. Also, let $\mathbb{L}^{\infty-}=\bigcap_{1<p<\infty} \mathbb{L}^{p}, \mathbb{D}_{\alpha}^{\infty-}=\bigcap_{1<p<\infty} \mathbb{D}_{\alpha}^{p}$ and $\mathbb{D}_{\infty}^{\infty-}=\bigcap_{1<p<\infty} \bigcap_{\alpha>0} \mathbb{D}_{\alpha}^{p}$. If we consider, more generally, $E$-valued functionals, $E$ being a real separable Hilbert space, the corresponding spaces are denoted by $\mathbb{L}^{p}(E), \mathbb{D}_{\alpha}^{p}(E)$, etc.

In the present paper we will suppose that the separable Hilbert space $H$ is an $\mathbb{L}^{2}$ space of the form $H=\mathbb{L}^{2}\left([0, T], \mathbb{R}^{d}\right)$. In order to avoid confusion we will use $D$ for the Malliavin derivatives and $\nabla$ for the classical derivatives or Fréchet derivatives of functions. We denote by $C_{p}^{\infty}\left(\mathbb{R}^{d}\right)$ be the set of all infinitely continuously differential function $f: \mathbb{R}^{d} \rightarrow \mathbb{R}$ such that $f$ and all of its partial derivatives have polynomial growth.

In the Malliavin calculus, a key role is played by the Malliavin covariance matrix which is defined as follows.

Definition 2.1. Suppose that $F=\left(F_{1}, \ldots, F_{d}\right)$ is a random vector whose components belong to the space $\mathbb{D}_{1}^{\infty-}$. We associate with $F$ the following random symmetric non-negative definite matrix:

$$
\Sigma_{F}(\omega)=\left(\sigma_{F}^{i j}(\omega)\right)_{1 \leqslant i, j \leqslant d}:=\left(\left\langle D F_{i}, D F_{j}\right\rangle_{H}\right)_{1 \leqslant i, j \leqslant d} .
$$

In one dimension setting,

$$
\Sigma_{F}(\omega)=\int_{0}^{T}\left(D_{r} F\right)^{2} d r
$$

The matrix $\Sigma(\omega)$ will be called the Malliavin covariance matrix of the random vector $F$. We will say that a random vector $F=\left(F_{1}, \ldots, F_{d}\right)$ whose components are in $\mathbb{D}_{\infty}^{\infty-}$ is non-degenerate if the Malliavin covariance matrix $\Sigma(\omega)$ is invertible almost surely and

$$
\Gamma_{F}(\omega)=\left(\gamma_{F}^{i j}(\omega)\right)_{1 \leqslant i, j \leqslant d}:=(\Sigma(\omega))^{-1} \in \mathbb{L}^{\infty-}\left(\mathbb{R}^{d} \otimes \mathbb{R}^{d}\right) .
$$

An important component in the study of the density of $F$ is the integration by parts formula. Here we give a local version of the integration by parts formula which can be seen in [4, Proposition 4.7].

Proposition 2.2. Suppose that:

(i) $F \in \mathbb{D}_{\infty}^{\infty-}\left(\mathbb{R}^{d}\right)$;

(ii) $G \in \mathbb{D}_{\infty}^{\infty-}$, and for some multi-index $\alpha, \Sigma_{F}$ is invertible almost surely on the set

$$
\{G \neq 0\} \bigcup_{\beta \leqslant \alpha}\left\{D^{\beta} G \neq 0\right\},
$$

and that, for any $p \neq 1$,

$$
E\left[\left|\operatorname{det}\left(\Sigma_{F}\right)\right|^{-p} 1_{\{G \neq 0\} \bigcup_{\beta \leqslant \alpha}\left\{D^{\beta} G \neq 0\right\}}\right]<\infty .
$$

Then for all $\phi \in C_{p}^{\infty}\left(\mathbb{R}^{d}\right)$, we have

$$
E\left[\left(\frac{\partial^{|\alpha|} \phi}{\partial y^{\alpha}} \circ F\right) G\right]=E\left[(\phi \circ F) H_{\alpha}(F, G) 1_{\{G \neq 0\} \bigcup_{\beta \leqslant \alpha}\left\{D^{\beta} G \neq 0\right\}}\right],
$$

where the elements $H_{\alpha}$ are recursively given by

$$
\begin{aligned}
H_{(i)}(F, G) & =H_{i}(F, G) \\
& :=-\sum_{j=1}^{d}\left\{\gamma_{i j} L F_{j} G+\left(D \gamma_{i j}, D F_{j}\right)_{H} G+\gamma_{i j}\left(D G, D F_{j}\right)\right\} \\
H_{\alpha}(F, G) & =H_{\alpha, \ldots, \alpha_{k}}(F, G) \\
& :=H_{\alpha_{k}}\left(F, H_{\alpha_{1}, \ldots, \alpha_{k-1}}(F, G)\right) .
\end{aligned}
$$


Moreover, for any $p>1$ and any multi-index $\alpha$, there exists a $C(p, \alpha)>0$ and integer $K(p, \alpha), M(p, \alpha), M^{\prime}(p, \alpha), N(p, \alpha), N^{\prime}(p, \alpha)$, such that, for any measurable set $A \subset\{G \neq 0\}$ $\bigcup_{\beta \leqslant \alpha}\left\{D^{\beta} G \neq 0\right\}$ and any $F, G$ as above, we have

$$
\begin{aligned}
E\left[\left|H_{\alpha}(F, G)\right|^{p} 1_{A}\right]^{1 / p} \leqslant & C(p, \alpha)\left\|\operatorname{det}\left(\Sigma_{F}\right)^{-1} 1_{A}\right\|_{K(p, \alpha)} \\
& \cdot\|G\|_{N(p, \alpha), M(p, \alpha)} \cdot\|F\|_{N^{\prime}(p, \alpha), M^{\prime}(p, \alpha)} .
\end{aligned}
$$

The following notation is taken from $[\mathbf{1}]$. Let $C([-\tau, 0], \mathbb{R})$ denote the Banach space of continuous paths $\eta:[-\tau, 0] \rightarrow \mathbb{R}$, furnished with the supremum norm

$$
\|\eta\|_{C}:=\sup _{-\tau \leqslant s \leqslant 0}|\eta(s)| .
$$

The symbol $H^{1, \infty}([-\tau, 0], \mathbb{R})$ denotes the Banach space of all continuous paths $\eta:[-\tau, 0] \rightarrow \mathbb{R}$, which are almost everywhere differentiable on $[-\tau, 0]$ and such that

$$
\operatorname{esssup}_{s \in[-\tau, 0]}\left|\eta^{\prime}(s)\right|<\infty \text {. }
$$

The space $H^{1, \infty}([-\tau, 0], \mathbb{R})$ is furnished with the $H^{1, \infty}$-norm

$$
\|\eta\|_{1, \infty}:=\sup _{-\tau \leqslant s \leqslant 0}|\eta(s)|+\operatorname{esssup}_{-\tau \leqslant s \leqslant 0}\left|\eta^{\prime}(s)\right| .
$$

The corresponding Banach space $C\left([-\tau, 0], \mathbb{R}^{d}\right)$ and $H^{1, \infty}\left([-\tau, 0], \mathbb{R}^{d}\right)$ of $\mathbb{R}^{d}$-valued mapping are defined analogously.

Let us end this section by introducing the Euler scheme for SDDEs. For simplicity of exposition, we will focus on one-dimensional SDDEs with a single delay and driven by a single Wiener process. The appropriate extensions of our analysis to higher dimension are straightforward. They are indicated in $\S 5$ of this paper. To further simplify the presentation, we deal with the SDDEs without drift, and we can easily extent our results to the general SDDEs under some appropriate conditions. More precisely, consider the one-dimensional SDDE for $\sigma \leqslant t \leqslant T$

$$
x(t)=\eta(0)+\int_{\sigma}^{t} g(x(u-\tau), x(u)) d W(u),
$$

with initial condition

$$
\eta(t-\sigma), \quad \sigma-\tau \leqslant t<\sigma,
$$

where $T>0$ is fixed, the initial instant $\sigma \in[0, T]$, the coefficient $g: \mathbb{R}^{2} \rightarrow \mathbb{R}$ satisfies suitable regularity and linear growth hypotheses, and the initial path $\eta \in H^{1, \infty}([-\tau, 0], \mathbb{R})$.

Let

$$
\pi:=\left\{-\tau=t_{-M}<t_{-M+1}<\ldots<t_{-1}<t_{0}=0<t_{1}<t_{2}<\ldots<t_{N-1}<t_{N}=T\right\}
$$

be a partition of $[-\tau, T]$, with equal size denoted by $|\pi|:=(T+\tau) /(N+M)$. Let $\sigma \in[0, T]$, and for any $u \in[\sigma, T]$, define $[u]:=t_{i-1} \vee \sigma$ whenever $u \in\left[t_{i-1}, t_{i}\right] \cap[\sigma, T]$. For each initial path $\eta \in H^{1, \infty}([-\tau, 0], \mathbb{R})$, define its piecewise-linear approximation $\eta^{\pi} \in H^{1, \infty}([-\tau, 0], \mathbb{R})$ by

$$
\eta^{\pi}(s):=\frac{\eta\left(t_{i}\right)-\eta\left(t_{i-1}\right)}{t_{i}-t_{i-1}}\left(s-t_{i-1}\right)+\eta\left(t_{i-1}\right)
$$

for $s \in\left[t_{i-1}, t_{i}\right),-M+1 \leqslant i \leqslant 0$, and $\eta^{\pi}(0):=\eta(0)$. Define the continuous Euler approximations $y:[\sigma-\tau, T] \times \Omega \rightarrow \mathbb{R}$ of the solution $x:[\sigma-\tau, T] \times \rightarrow \mathbb{R}$ of (3) to be solutions of the SDDEs:

$$
y(t)= \begin{cases}\eta(0)+\int_{\sigma}^{t} g\left(y\left([u]-\tau_{2}\right), y([u])\right) d W(u), & t \geqslant \sigma, \\ \eta(t-\sigma), & \sigma-\tau<t<\sigma .\end{cases}
$$

Denote the solutions of (3) and (4) by $x(t ; \sigma, \eta)$ and $y(t ; \sigma, \eta), \sigma-\tau \leqslant t \leqslant T$. 


\section{Main result}

We shall make use of the following assumptions.

(A.I) The function $g: \mathbb{R}^{2} \rightarrow \mathbb{R}$ is a $C^{\infty}$ function, whose derivatives of any order are bounded (but it is not assumed to be bounded itself).

(A.II) For any $t \in[0, T], x(t ; 0, \eta)$ is non-degenerate, that is, the inverse Malliavin covariance matrix $\gamma_{x(t ; 0, \eta)}:=\left(\int_{0}^{T}\left(D_{r} x(t ; 0, \eta)\right)^{2} d r\right)^{-1}$ satisfies

$$
\gamma_{x(t ; 0, \eta)} \in \mathbb{L}^{\infty-} .
$$

REMARK 3.1. The condition (5) is satisfied in the uniformly elliptic case, that is $g(x) \geqslant \varepsilon>0$ for all $x \in \mathbb{R}$ (see [10]), or under some weaker assumptions (see [2]).

Theorem 3.2. Let $x(\cdot ; \sigma, \eta)$ be the unique solution of (3) with initial path $\eta \in$ $H^{1, \infty}([-\tau, 0], \mathbb{R})$. Suppose that the coefficient $g$ satisfies the assumption (A.I) and $x(t ; 0, \eta)$ satisfies the assumption (A.II). Let $\pi$ be a partition of $[-\tau, T]$ with equal size $|\pi|$, and $\eta^{\pi} \in$ $H^{1, \infty}([-\tau, 0], \mathbb{R})$ be the piecewise-linear approximation of $\eta$ along the partition $\pi$. Denote by $y(\cdot ; \sigma, \eta)$ the Euler approximation to $x(\cdot ; \sigma, \eta)$ associated with the partition $\pi$ and defined by (4). Then for a bounded measurable function $\phi: \mathbb{R} \rightarrow \mathbb{R}$, there exists a positive constant $K$ and a positive integer $q$ such that

$$
\left|E \phi(x(t ; \sigma, \eta))-E \phi\left(y\left(t ; \sigma, \eta^{\pi}\right)\right)\right| \leqslant K\left(1+\|\eta\|_{1, \infty}^{q}\right)|\pi|
$$

for all $t \in[\sigma-\tau, T], \sigma \in[0, T]$ and all $\eta \in H^{1, \infty}([-\tau, 0], \mathbb{R})$. The constant $K$ may depend on $T, q$ and the test function $\phi$, but is independent of $\pi, \eta, t \in[\sigma, T]$ and $\sigma \in[0, T]$.

REMARK 3.3. The boundedness hypothesis on $\phi$ can be relaxed: the preceding technique can be improved to deal with the case of functions $\phi$ which are measurable and have polynomial growth. The extension is verified along the same lines as in [3, Section 6$]$.

REMARK 3.4. The above theorem can be easily extended to higher dimensions, and we put the detailed extension in $\S 5$.

\section{Proof of Theorem 3.2}

We first state a technical lemma which will paly an important role in the proof of Theorem 3.2.

Lemma 4.1. Suppose that the coefficient $g$ satisfies the assumption (A.I). Let $x(\cdot ; \sigma, \eta)$ be the unique solution of $(3)$ with initial path $\eta \in \mathbb{L}^{\infty-}\left(\Omega, C([-\tau, 0], \mathbb{R}) ; \mathscr{F}_{\sigma}\right)$ which are Malliavin smooth and such that

$$
\sup _{\sigma-\tau \leqslant s_{1}, \ldots, s_{k} \leqslant \sigma} E\left\|D_{s_{1}} D_{s_{2}} \ldots D_{s_{k}} \eta\right\|_{\infty}^{p}<\infty
$$

for every integer $k \geqslant 1$ and any $p \geqslant 1$. Let $\pi$ be a partition of $[-\tau, T]$ with equal size $|\pi|$. Denote by $y(\cdot ; \sigma, \eta)$ the Euler approximation to $x(\cdot ; \sigma, \eta)$ associated with the partition $\pi$ and defined by (4). Then for any $p>1$ and $k \geqslant 1$, there exists a positive constant $C$ such that

$$
\begin{aligned}
\|x(t ; \sigma, \eta)-y(t ; \sigma, \eta)\|_{k, p} \leqslant & C\left(1+E\|\eta\|_{C}^{2^{k} p}+\sup _{\sigma-\tau \leqslant s \leqslant \sigma} E\left\|D_{s} \eta\right\|_{\infty}^{2^{k} p}\right. \\
& \left.+\ldots+\sup _{\sigma-\tau \leqslant s_{1}, \ldots, s_{k} \leqslant \sigma} E\left\|D_{s_{1}} D_{s_{2}} \ldots D_{s_{k}} \eta\right\|_{\infty}^{2^{k} p}\right)|\pi|^{1 / 2} .
\end{aligned}
$$

Proof. This result actually follows from a general theorem of He, Ren and Zhang [16, Proposition 4.8], only now one has to do a little bookkeeping along the way. 
The next lemma states the Markov property for the segment $x_{t}$ and $y_{t}$.

Lemma 4.2. Let $x(\cdot ; \sigma, \eta)$ and $y(\cdot ; \sigma, \eta)$ be as in the statement of Theorem 3.2. Let $t \in[\sigma, T]$ and

$$
\pi:=t_{-M}<t_{-M+1}<\ldots<t_{-1}<t_{0}=0<t_{1}<t_{2}<\ldots<t_{N-1}<t_{N}=T
$$

be a partition of $[-\tau, T]$ with equal size $|\pi|=(T+\tau) /(N+M)$. Without loss of generality assume that $\sigma=0$ and $t=t_{n} \in \pi$ for some $0 \leqslant n \leqslant N$. Then we have

$$
\begin{aligned}
& y\left(t_{n} ; t_{i}, \lambda x_{t_{i}}\left(\cdot ; t_{i-1}, x_{t_{i-1}}(\cdot ; 0, \eta)\right)+(1-\lambda) y_{t_{i}}\left(\cdot ; t_{i-1}, x_{t_{i-1}}(\cdot ; 0, \eta)\right)\right) \\
& \quad=\lambda y\left(t_{n} ; t_{i}, x_{t_{i}}(\cdot ; 0, \eta)\right)+(1-\lambda) y\left(t_{n} ; t_{i-1}, x_{t_{i-1}}(\cdot ; 0, \eta)\right) .
\end{aligned}
$$

In particular, we have

$$
\begin{gathered}
y\left(t_{n} ; t_{i}, x_{t_{i}}\left(\cdot ; t_{i-1}, x_{t_{i-1}}(\cdot ; 0, \eta)\right)\right)=y\left(t_{n} ; t_{i}, x_{t_{i}}(\cdot ; 0, \eta)\right), \\
y\left(t_{n} ; t_{i}, y_{t_{i}}\left(\cdot ; t_{i-1}, x_{t_{i-1}}(\cdot ; 0, \eta)\right)\right)=y\left(t_{n} ; t_{i-1}, x_{t_{i-1}}(\cdot ; 0, \eta)\right) .
\end{gathered}
$$

Proof. Using the Markov property for the segments $x_{t}$ and $y_{t}(\mathrm{cf} .[\mathbf{1 2}, \mathbf{1 3}])$, we have

$$
\begin{aligned}
y\left(t_{n} ;\right. & \left.t_{i}, \lambda x_{t_{i}}\left(\cdot ; t_{i-1}, x_{t_{i-1}}(\cdot ; 0, \eta)\right)+(1-\lambda) y_{t_{i}}\left(\cdot ; t_{i-1}, x_{t_{i-1}}(\cdot ; 0, \eta)\right)\right) \\
= & \lambda x_{t_{i}}\left(0 ; t_{i-1}, x_{t_{i-1}}(\cdot ; 0, \eta)\right)+(1-\lambda) y_{t_{i}}\left(0 ; t_{i-1}, x_{t_{i-1}}(\cdot ; 0, \eta)\right) \\
& +\int_{t_{i}}^{t_{n}} g(y([u]-\tau), y([u])) d W(u) \\
= & \lambda x_{t_{i}}(0 ; 0, \eta)+(1-\lambda)\left[x_{t_{i-1}}(0 ; 0, \eta)+\int_{t_{i-1}}^{t_{i}} g\left(y\left(t_{i-1}-\tau\right), y\left(t_{i-1}\right)\right) d W(u)\right] \\
& +\int_{t_{i}}^{t_{n}} g(y([u]-\tau), y([u])) d W(u) \\
= & \lambda\left[x_{t_{i}}(0 ; 0, \eta)+\int_{t_{i}}^{t_{n}} g(y([u]-\tau), y([u])) d W(u)\right]+(1-\lambda)\left[x_{t_{i-1}}(0 ; 0, \eta)\right. \\
& \left.+\int_{t_{i-1}}^{t_{n}} g(y([u]-\tau), y([u])) d W(u)\right] \\
= & \lambda y\left(t_{n} ; t_{i}, x_{t_{i}}(\cdot ; 0, \eta)\right)+(1-\lambda) y\left(t_{n} ; t_{i-1}, x_{t_{i-1}}(\cdot ; 0, \eta)\right) .
\end{aligned}
$$

The proof is now completed.

The proof of Theorem 3.2 also requires the following sequence of lemmas. The following lemma establishes the tame character of the Euler approximation $y(t ; \sigma, \eta)$ and its Fréchet derivative $\nabla y(t ; \sigma, \eta)$ in the initial path. The detailed proof is essentially contained in [1, Lemma 3.2].

Lemma 4.3. Let $g, x(\cdot ; \sigma, \eta)$ and $y(\cdot ; \sigma, \eta)$ be as in the statement of Theorem 3.2. Fix a partition point $t_{i} \in \pi$ for some $i \in 0,1, \ldots, N$. Then for almost every $\omega \in \Omega$, the function

$$
\begin{aligned}
{\left[t_{i}, T\right] \times C([-\tau, 0], \mathbb{R}) } & \rightarrow \mathbb{R}, \\
(t, \eta) & \mapsto y\left(t, \omega ; t_{i}, \eta\right)
\end{aligned}
$$

is a tame function. That is to say, there exists a deterministic function $F: \mathbb{R}^{+} \times \mathbb{R}^{k+1} \times \mathbb{R}^{h} \times$ $\mathbb{R}^{l} \rightarrow \mathbb{R}$ which is piecewise continuous in the first variable (the time variable) and of class $C_{b}^{\infty}$ in all other variables (space variables), and there exist fixed numbers $t_{1}, t_{2}, \ldots, t_{k} \leqslant t$, $s_{1}, s_{2}, \ldots, s_{h} \leqslant t, \mu_{1}, \mu_{2}, \ldots, \mu_{l} \in[-\tau, 0]$ such that almost everywhere

$$
y\left(t ; t_{i}, \eta\right)=F\left(t, W(t), W\left(t_{1}\right), \ldots, W\left(t_{k}\right), s_{1}, s_{2}, \ldots, s_{h}, \eta\left(\mu_{1}\right), \ldots, \eta\left(\mu_{l}\right)\right)
$$


for all $\eta \in C([-\tau, 0], \mathbb{R})$ and all $t \in\left[t_{i}, T\right]$. In particular, for almost every $\omega \in \Omega$ and each $t \in\left[t_{i}, T\right]$, the map

$$
C([-\tau, 0], \mathbb{R}) \ni \eta \mapsto y\left(t, \omega ; t_{i}, \eta\right) \in \mathbb{R}
$$

is $C^{\infty}$ in the Fréchet sense, and for all $\eta \in C([-\tau, 0], \mathbb{R})$ and every bounded measurable function $\xi:[-\tau, 0] \rightarrow \mathbb{R}$,

$$
\begin{aligned}
\nabla y\left(t, \omega ; t_{i}, \eta\right)(\xi)= & \sum_{m=1}^{l} \partial_{m} F\left(t, W(t, \omega), W\left(t_{1}, \omega\right), \ldots, W\left(t_{k}, \omega\right), s_{1}, \ldots, s_{h},\right. \\
& \left.\eta\left(\mu_{1}\right), \ldots, \eta\left(\mu_{m}\right), \ldots, \eta\left(\mu_{l}\right)\right) \xi\left(\mu_{m}\right)
\end{aligned}
$$

where $\partial_{m} F$ denotes the partial derivative of $F$ with respect to the variable $\eta\left(\mu_{m}\right)$.

The following lemma is key to the proof of Theorem 3.2.

Lemma 4.4. Let $g, x(\cdot ; \sigma, \eta)$ and $y(\cdot ; \sigma, \eta)$ be as in the statement of Theorem 3.2. Fix $\eta \in C([-\tau, 0], \mathbb{R})$ of bounded variation. For each $1 \leqslant i \leqslant N$, define the process $\Lambda^{i}:[-\tau, 0] \times \Omega \rightarrow$ $\mathbb{R}$ by

$$
\Lambda^{i}:=x_{t_{i}}\left(\cdot ; t_{i-1}, x_{t_{i-1}}(\cdot ; 0, \eta)\right)-y_{t_{i}}\left(\cdot ; t_{i-1}, x_{t_{i-1}}(\cdot ; 0, \eta)\right), \quad s \in[-\tau, 0] .
$$

For brevity of notation, set $x(u):=x(u ; 0, \eta)$ and $y(u):=y(u ; 0, \eta)$ for $u \in[-\tau, T]$. Then we have

$$
\begin{aligned}
\Lambda^{i}(s) & =\int_{t_{i-1}}^{\left(t_{i}+s\right) \vee t_{i-1}}[g(x(u-\tau), x(u))-g(x([u]-\tau), x([u]))] d W(u) \\
& :=\sum_{j=1}^{5} \Lambda_{j}^{i}(s), \quad s \in[-\tau, 0]
\end{aligned}
$$

where

$$
\begin{aligned}
\Lambda_{1}^{i}(s):= & \int_{t_{i-1}}^{\left(t_{i}+s\right) \vee t_{i-1}} \int_{[u]}^{u} \frac{\partial g}{\partial x_{1}}(x(v-\tau), x(v)) 1_{[0, \tau)}(v) d \eta\left(v-\tau_{2}\right) d W(u), \\
\Lambda_{2}^{i}(s):= & \int_{t_{i-1}}^{\left(t_{i}+s\right) \vee t_{i-1}} \int_{[u]}^{u} \frac{\partial g}{\partial x_{1}}(x(v-\tau), x(v)) g(x(v-2 \tau), x(v-\tau)) 1_{[\tau, \infty)}(v) d W(v-\tau) d W(u) \\
& +\int_{t_{i-1}}^{\left(t_{i}+s\right) \vee t_{i-1}} \int_{[u]}^{u} \frac{\partial g}{\partial x_{2}}(x(v-\tau), x(v)) g(x(v-\tau), x(v)) d W(v) d W(u), \\
\Lambda_{3}^{i}(s):= & \int_{t_{i-1}}^{\left(t_{i}+s\right) \vee t_{i-1}} \int_{[u]}^{u} \frac{\partial^{2} g}{\partial x_{1} \partial x_{2}}(x(v-\tau), x(v)) \\
& \times g(x(v-2 \tau), x(v-\tau)) 1_{[\tau, \infty)}(v) D_{v-\tau} x(v) d v d W(u), \\
\Lambda_{4}^{i}(s):= & \frac{1}{2} \int_{t_{i-1}}^{\left(t_{i}+s\right) \vee t_{i-1}} \int_{[u]}^{u} \frac{\partial^{2} g}{\partial x_{1}^{2}}(x(v-\tau), x(v)) g(x(v-2 \tau), x(v-\tau))^{2} 1_{[\tau, \infty)}(v) d v d W(u), \\
\Lambda_{5}^{i}(s):= & \frac{1}{2} \int_{t_{i-1}}^{\left(t_{i}+s\right) \vee t_{i-1}} \int_{[u]}^{u} \frac{\partial^{2} g}{\partial x_{2}^{2}}(x(v-\tau), x(v)) g(x(v-\tau), x(v))^{2} d v d W(u),
\end{aligned}
$$


and

$$
D_{r} \Lambda^{i}(s):=\sum_{j=1}^{19} \Xi_{j}^{i}(s, r), \quad s \in[-\tau, 0], r \in[0, T]
$$

where

$$
\begin{aligned}
\Xi_{1}^{i}(s, r):= & \int_{t_{i-1}}^{\left(t_{i}+s\right) \vee t_{i-1}} \int_{[u]}^{u} \frac{\partial^{2} g}{\partial x_{1}^{2}}(x(v-\tau), x(v)) 1_{[0, \tau)}(v) D_{r} x(u) d \eta\left(v-\tau_{2}\right) d W(u), \\
\Xi_{2}^{i}(s, r):= & \int_{t_{i-1}}^{\left(t_{i}+s\right) \vee t_{i-1}} \int_{[u]}^{u} \frac{\partial^{2} g}{\partial x_{1}^{2}}(x(v-\tau), x(v)) g(x(v-2 \tau), x(v-\tau)) \\
& \times 1_{[\tau, \infty)}(v) D_{r} x(u) d W(v-\tau) d W(u) \\
& +\int_{t_{i-1}}^{\left(t_{i}+s\right) \vee t_{i-1}} \int_{[u]}^{u} \frac{\partial^{2} g}{\partial x_{1} \partial x_{2}}(x(v-\tau), x(v)) g(x(v-\tau), x(v)) D_{r} x(u) d W(v) d W(u),
\end{aligned}
$$$$
\Xi_{3}^{i}(s, r):=\int_{t_{i-1}}^{\left(t_{i}+s\right) \vee t_{i-1}} \int_{[u]}^{u} \frac{\partial^{3} g}{\partial x_{1}^{2} \partial x_{2}}(x(v-\tau), x(v)) g(x(v-2 \tau), x(v-\tau))
$$$$
\times 1_{[\tau, \infty)}(v) D_{v-\tau} x(v) D_{r} x(u) d v d W(u),
$$$$
\Xi_{4}^{i}(s, r):=\frac{1}{2} \int_{t_{i-1}}^{\left(t_{i}+s\right) \vee t_{i-1}} \int_{[u]}^{u} \frac{\partial^{3} g}{\partial x_{1}^{3}}(x(v-\tau), x(v)) g(x(v-2 \tau), x(v-\tau))^{2}
$$$$
\times 1_{[\tau, \infty)}(v) D_{r} x(u) d v d W(u),
$$$$
\Xi_{5}^{i}(s, r):=\frac{1}{2} \int_{t_{i-1}}^{\left(t_{i}+s\right) \vee t_{i-1}} \int_{[u]}^{u} \frac{\partial^{3} g}{\partial x_{1} \partial x_{2}^{2}}(x(v-\tau), x(v)) g(x(v-\tau), x(v))^{2}
$$$$
\times 1_{[\tau, \infty)}(v) D_{r} x(u) d v d W(u),
$$$$
\Xi_{6}^{i}(s, r):=\int_{t_{i-1}}^{\left(t_{i}+s\right) \vee t_{i-1}} \int_{[u]}^{u} \frac{\partial^{2} g}{\partial x_{1} \partial x_{2}}(x(v-\tau), x(v)) 1_{[0, \tau)}(v) D_{r} x(u-\tau) d \eta\left(v-\tau_{2}\right) d W(u),
$$$$
\Xi_{7}^{i}(s, r):=\int_{t_{i-1}}^{\left(t_{i}+s\right) \vee t_{i-1}} \int_{[u]}^{u} \frac{\partial^{2} g}{\partial x_{1} \partial x_{2}}(x(v-\tau), x(v)) g(x(v-2 \tau), x(v-\tau))
$$$$
\times 1_{[\tau, \infty)}(v) D_{r} x(u-\tau) d W(v-\tau) d W(u)
$$$$
+\int_{t_{i-1}}^{\left(t_{i}+s\right) \vee t_{i-1}} \int_{[u]}^{u} \frac{\partial^{2} g}{\partial x_{2}^{2}}(x(v-\tau), x(v)) g(x(v-\tau), x(v)) D_{r} x(u-\tau) d W(v) d W(u),
$$

$$
\begin{aligned}
\Xi_{8}^{i}(s, r):= & \int_{t_{i-1}}^{\left(t_{i}+s\right) \vee t_{i-1}} \int_{[u]}^{u} \frac{\partial^{3} g}{\partial x_{1} \partial x_{2}^{2}}(x(v-\tau), x(v)) \\
& \times g(x(v-2 \tau), x(v-\tau)) 1_{[\tau, \infty)}(v) D_{v-\tau} x(v) d v d W(u),
\end{aligned}
$$




$$
\begin{aligned}
& \Xi_{9}^{i}(s, r):=\frac{1}{2} \int_{t_{i-1}}^{\left(t_{i}+s\right) \vee t_{i-1}} \int_{[u]}^{u} \frac{\partial^{3} g}{\partial x_{1}^{2} \partial x_{2}}(x(v-\tau), x(v)) g(x(v-2 \tau), x(v-\tau))^{2} \\
& \times 1_{[\tau, \infty)}(v) D_{r} x(u-\tau) d v d W(u), \\
& \Xi_{10}^{i}(s, r):=\frac{1}{2} \int_{t_{i-1}}^{\left(t_{i}+s\right) \vee t_{i-1}} \int_{[u]}^{u} \frac{\partial^{3} g}{\partial x_{2}^{3}}(x(v-\tau), x(v)) g(x(v-\tau), x(v))^{2} D_{r} x(u-\tau) d v d W(u), \\
& \Xi_{11}^{i}(s, r):=\int_{[r]}^{r} \frac{\partial g}{\partial x_{1}}(x(v-\tau), x(v)) 1_{[0, \tau)}(v) d \eta(v-\tau) 1_{\left(t_{i-1},\left(t_{i}+s\right) \vee t_{i-1}\right]}(r), \\
& \Xi_{12}^{i}(s, r):=\int_{[r]}^{r} \frac{\partial g}{\partial x_{1}} g(x(v-2 \tau), x(v-\tau)) 1_{[\tau, \infty)}(v) d W(v-\tau) 1_{\left(t_{i-1},\left(t_{i}+s\right) \vee t_{i-1}\right]}(r) \\
& +\int_{[r]}^{r} \frac{\partial g}{\partial x_{2}} g(x(v-\tau), x(v)) d W(v) 1_{\left(t_{i-1},\left(t_{i}+s\right) \vee t_{i-1}\right]}(r), \\
& \Xi_{13}^{i}(s, r):=\int_{[r]}^{r} \frac{\partial^{2} g}{\partial x_{1} \partial x_{2}}(x(v-\tau), x(v)) g(x(v-2 \tau), x(v-\tau)) \\
& \times 1_{[\tau, \infty)}(v) D_{v-\tau} x(v) d v 1_{\left(t_{i-1},\left(t_{i}+s\right) \vee t_{i-1}\right]}(r), \\
& \Xi_{14}^{i}(s, r):=\frac{1}{2} \int_{[r]}^{r} \frac{\partial^{2} g}{\partial x_{1}^{2}}(x(v-\tau), x(v)) g(x(v-2 \tau), x(v-\tau))^{2} \\
& \times 1_{\left[\tau_{2}, \infty\right)}(v) d v 1_{\left(t_{i-1},\left(t_{i}+s\right) \vee t_{i-1}\right]}(r), \\
& \Xi_{15}^{i}(s, r):=\frac{1}{2} \int_{[r]}^{r} \frac{\partial^{2} g}{\partial x_{2}^{2}}(x(v-\tau), x(v)) g(x(v-\tau), x(v))^{2} d v 1_{\left(t_{i-1},\left(t_{i}+s\right) \vee t_{i-1}\right]}(r), \\
& \Xi_{16}^{i}(s, r):=\int_{t_{i-1}}^{\left(t_{i}+s\right) \vee t_{i-1}} g(x(r-\tau), x(r)) 1_{([u]-\tau, u-\tau]}(r) 1_{\tau<[u]} \frac{\partial}{\partial x_{1}} g(x([u]-\tau), x([u])) d W(u) \\
& +\int_{t_{i-1}}^{\left(t_{i}+s\right) \vee t_{i-1}} g(x(r-\tau), x(r)) 1_{(0, u-\tau]}(r) 1_{[u] \leqslant \tau<u} \frac{\partial}{\partial x_{1}} g(x([u]-\tau), x([u])) d W(u) \\
& +\int_{t_{i-1}}^{\left(t_{i}+s\right) \vee t_{i-1}} g(x(r-\tau), x(r)) 1_{([u], u]}(r) \frac{\partial}{\partial x_{2}} g(x([u]-\tau), x([u])) d W(u), \\
& \Xi_{17}^{i}(s, r):=\int_{t_{i-1}}^{\left(t_{i}+s\right) \vee t_{i-1}} \int_{[u]-\tau}^{u-\tau} \frac{\partial}{\partial x_{1}} g(x(v-\tau), x(v)) D_{r} x(v-\tau) 1_{\tau<[u]} \\
& \times \frac{\partial}{\partial x_{1}} g(x([u]-\tau), x([u])) d W(v) d W(u) \\
& +\int_{t_{i-1}}^{\left(t_{i}+s\right) \vee t_{i-1}} \int_{[u]-\tau}^{u-\tau} \frac{\partial}{\partial x_{2}} g(x(v-\tau), x(v)) D_{r} x(v) 1_{\tau<[u]} \\
& \times \frac{\partial}{\partial x_{1}} g(x([u]-\tau), x([u])) d W(v) d W(u),
\end{aligned}
$$




$$
\begin{aligned}
\Xi_{18}^{i}(s, r):= & \int_{t_{i-1}}^{\left(t_{i}+s\right) \vee t_{i-1}} \int_{0}^{u-\tau} \frac{\partial}{\partial x_{1}} g(x(v-\tau), x(v)) D_{r} x(v-\tau) 1_{[u] \leqslant \tau<u} \\
& \times \frac{\partial}{\partial x_{1}} g(x([u]-\tau), x([u])) d W(v) d W(u) \\
& +\int_{t_{i-1}}^{\left(t_{i}+s\right) \vee t_{i-1}} \int_{0}^{u-\tau} \frac{\partial}{\partial x_{2}} g(x(v-\tau), x(v)) D_{r} x(v) 1_{[u] \leqslant \tau<u} \\
& \times \frac{\partial}{\partial x_{1}} g(x([u]-\tau), x([u])) d W(v) d W(u), \\
\Xi_{19}^{i}(s, r):= & \int_{t_{i-1}}^{\left(t_{i}+s\right) \vee t_{i-1}} \int_{[u]}^{u} \frac{\partial}{\partial x_{1}} g(x(v-\tau), x(v)) D_{r} x(v-\tau) \\
& \times \frac{\partial}{\partial x_{1}} g(x([u]-\tau), x([u])) d W(v) d W(u) \\
& +\int_{t_{i-1}}^{\left(t_{i}+s\right) \vee t_{i-1}} \int_{[u]}^{u} \frac{\partial}{\partial x_{2}} g(x(v-\tau), x(v)) D_{r} x(v) \\
& \times \frac{\partial}{\partial x_{2}} g(x([u]-\tau), x([u])) d W(v) d W(u) .
\end{aligned}
$$

Proof. Fix $1 \leqslant i \leqslant N$. Suppose that $t_{i-1} \leqslant u<t_{i}$. Then $[u]=t_{i-1}$ and $[u]-\tau=t_{i-1}-\tau<$ $t_{i-1}$. For the proof of the first assertion (13) of the lemma, the reader can see [1, Lemma 3.3]. Next we prove the second assertion (14) of the lemma. Taking the Malliavin derivative of $\Lambda^{i}(s)$, we have

$$
\begin{aligned}
D_{r} \Lambda^{i}(s)=[ & g(x(r-\tau), x(r))-g(x([r]-\tau), x([r]))] 1_{\left(t_{i-1},\left(t_{i}+s\right) \vee t_{i-1}\right]}(r) \\
& +\int_{t_{i-1}}^{\left(t_{i}+s\right) \vee t_{i-1}}\left[\frac{\partial}{\partial x_{1}} g(x(u-\tau), x(u)) D_{r} x(u-\tau)+\frac{\partial}{\partial x_{2}} g(x(u-\tau), x(u)) D_{r} x(u)\right. \\
& \left.-\frac{\partial}{\partial x_{1}} g(x([u]-\tau), x([u])) D_{r} x([u]-\tau)-\frac{\partial}{\partial x_{2}} g(x([u]-\tau), x([u])) D_{r} x([u])\right] d W(u) \\
= & g(x(r-\tau), x(r))-g(x([r]-\tau), x([r]))] 1_{\left(t_{i-1},\left(t_{i}+s\right) \vee t_{i-1}\right]}(r) \\
& +\int_{t_{i-1}}^{\left(t_{i}+s\right) \vee t_{i-1}}\left[\frac{\partial}{\partial x_{1}} g(x(u-\tau), x(u))-\frac{\partial}{\partial x_{1}} g(x([u]-\tau), x([u]))\right] D_{r} x(u-\tau) d W(u) \\
& +\int_{t_{i-1}}^{\left(t_{i}+s\right) \vee t_{i-1}}\left[D_{r} x(u-\tau)-D_{r} x([u]-\tau)\right] \frac{\partial}{\partial x_{1}} g(x([u]-\tau), x([u])) d W(u) \\
& +\int_{t_{i_{-1}}}^{\left(t_{i}+s\right) \vee t_{i-1}}\left[\frac{\partial}{\partial x_{2}} g(x(u-\tau), x(u))-\frac{\partial}{\partial x_{2}} g(x([u]-\tau), x([u]))\right] D_{r} x(u) d W(u) \\
& +\int_{t_{i-1}}^{\left(t_{i}+s\right) \vee t_{i-1}}\left[D_{r} x(u)-D_{r} x([u])\right] \frac{\partial}{\partial x_{2}} g(x([u]-\tau), x([u])) d W(u) .
\end{aligned}
$$


Applying the tame Itô formula which can been found in [1, Theorem 2.1], we obtain

$$
\begin{aligned}
& g(x(r-\tau), x(r))-g(x([r]-\tau), x([r])) \\
& =\int_{[r]}^{r} \frac{\partial g}{\partial x_{1}}(x(v-\tau), x(v)) 1_{[0, \tau)}(v) d \eta(v-\tau) \\
& \quad+\int_{[r]}^{r} \frac{\partial g}{\partial x_{1}} g(x(v-2 \tau), x(v-\tau)) 1_{[\tau, \infty)}(v) d W(v-\tau) \\
& \quad+\int_{[r]}^{r} \frac{\partial g}{\partial x_{2}} g(x(v-\tau), x(v)) d W(v) \\
& \quad+\int_{[r]}^{r} \frac{\partial^{2} g}{\partial x_{1} \partial x_{2}}(x(v-\tau), x(v)) g(x(v-2 \tau), x(v-\tau)) 1_{[\tau, \infty)}(v) D_{v-\tau} x(v) d v \\
& \quad+\frac{1}{2} \int_{[r]}^{r} \frac{\partial^{2} g}{\partial x_{1}^{2}}(x(v-\tau), x(v)) g(x(v-2 \tau), x(v-\tau))^{2} 1_{\left[\tau_{2}, \infty\right)}(v) d v \\
& \quad+\frac{1}{2} \int_{[r]}^{r} \frac{\partial^{2} g}{\partial x_{2}^{2}}(x(v-\tau), x(v)) g(x(v-\tau), x(v))^{2} d v .
\end{aligned}
$$

Similarly, it follows that

$$
\frac{\partial}{\partial x_{1}} g(x(u-\tau), x(u))-\frac{\partial}{\partial x_{1}} g(x([u]-\tau), x([u]))
$$

and

$$
\frac{\partial}{\partial x_{2}} g(x(u-\tau), x(u))-\frac{\partial}{\partial x_{2}} g(x([u]-\tau), x([u]))
$$

have the same forms as in (16). Taking the Malliavin derivatives of $x(u-\tau)-x([u]-\tau)$, we have

$$
\begin{aligned}
D_{r} x & (u-\tau)-D_{r} x([u]-\tau) \\
= & D_{r}\left(\int_{[u]-\tau}^{u-\tau} g(x(v-\tau), x(v)) d W(v)\right) 1_{\tau<[u]} \\
& +D_{r}\left(\eta(0)+\int_{0}^{u-\tau} g(x(v-\tau), x(v)) d W(v)-\eta([u]-\tau)\right) 1_{[u] \leqslant \tau<u} \\
& +D_{r}(\eta(u-\tau)-\eta([u]-\tau)) 1_{\tau \geqslant u} \\
= & {\left[g(x(r-\tau), x(r)) 1_{([u]-\tau, u-\tau]}(r)+\int_{[u]-\tau}^{u-\tau} \frac{\partial}{\partial x_{1}} g(x(v-\tau), x(v)) D_{r} x(v-\tau) d W(v)\right.} \\
& \left.+\int_{[u]-\tau}^{u-\tau} \frac{\partial}{\partial x_{2}} g(x(v-\tau), x(v)) D_{r} x(v) d W(v)\right] 1_{\tau<[u]} \\
& +\left[g(x(r-\tau), x(r)) 1_{(0, u-\tau]}(r)+\int_{0}^{u-\tau} \frac{\partial}{\partial x_{1}} g(x(v-\tau), x(v)) D_{r} x(v-\tau) d W(u)\right. \\
& \left.+\int_{0}^{u-\tau} \frac{\partial}{\partial x_{2}} g(x(v-\tau), x(v)) D_{r} x(v) d W(u)\right] 1_{[u] \leqslant \tau<u} .
\end{aligned}
$$


Similarly we have

$$
\begin{aligned}
D_{r} x(u)-D_{r} x([u])= & D_{r}\left(\int_{[u]}^{u} g(x(v-\tau), x(v)) d W(v)\right) \\
= & g(x(r-\tau), x(r)) 1_{([u], u]}(r) \\
& +\int_{[u]}^{u} \frac{\partial}{\partial x_{1}} g(x(v-\tau), x(v)) D_{r} x(v-\tau) d W(v) \\
& +\int_{[u]}^{u} \frac{\partial}{\partial x_{2}} g(x(v-\tau), x(v)) D_{r} x(v) d W(v) .
\end{aligned}
$$

Now substituting from (16)-(20) into (15), we complete the proof.

Lemma 4.5. Let $g, x(\cdot ; \sigma, \eta)$ and $y(\cdot ; \sigma, \eta)$ be as in the statement of Theorem 3.2. Then for every integer $k \geqslant 0$ and any $p \geqslant 1$,

$$
\begin{gathered}
\sup _{0 \leqslant \sigma \leqslant T} \sup _{\sigma-\tau \leqslant u, \ldots, u_{k}, t \leqslant T} E\left|D_{u_{1}} \ldots D_{u_{k}} x(t ; \sigma, \eta)\right|^{2 p} \\
\leqslant K\left(1+E\|\eta\|_{C}^{2^{k} p}+\sup _{\sigma-\tau \leqslant s \leqslant \sigma} E\left\|D_{s} \eta\right\|_{\infty}^{2^{k} p}+\ldots\right. \\
\left.+\sup _{\sigma-\tau \leqslant s_{1}, \ldots, s_{k} \leqslant \sigma} E\left\|D_{s_{1}} D_{s_{2}} \ldots D_{s_{k}} \eta\right\|_{\infty}^{2^{k} p}\right), \\
\sup _{0 \leqslant \sigma \leqslant T} \sup _{\sigma-\tau \leqslant u, \ldots, u_{k}, t \leqslant T} E\left|D_{u_{1}} \ldots D_{u_{k}} y(t ; \sigma, \eta)\right|^{2 p} \\
\leqslant K\left(1+E\|\eta\|_{C}^{2^{k} p}+\sup _{\sigma-\tau \leqslant s \leqslant \sigma} E\left\|D_{s} \eta\right\|_{\infty}^{2^{k} p}+\ldots\right. \\
\left.+\sup _{\sigma-\tau \leqslant s_{1}, \ldots, s_{k} \leqslant \sigma} E\left\|D_{s_{1}} D_{s_{2}} \ldots D_{s_{k}} \eta\right\|_{\infty}^{2^{k} p}\right)
\end{gathered}
$$

and

$$
\begin{aligned}
& \sup _{\substack{\|\xi\|_{\infty} \leqslant 1, \xi \in \mathbb{L}^{\infty}([-\tau, 0], \mathbb{R})}} \sup _{0 \leqslant \sigma \leqslant T} \sup _{\sigma-\tau \leqslant u_{1}, \ldots, u_{k}, t \leqslant T} E\left|D_{u_{1}} \ldots D_{u_{k}} \nabla y(t ; \sigma, \eta)(\xi)\right|^{2 p} \\
& \leqslant K\left(1+E\|\eta\|_{C}^{2^{k+1} p}+\sup _{\sigma-\tau \leqslant s \leqslant \sigma} E\left\|D_{s} \eta\right\|_{\infty}^{2^{k+1} p}\right. \\
&\left.+\ldots+\sup _{\sigma-\tau \leqslant s_{1}, \ldots, s_{k} \leqslant \sigma} E\left\|D_{s_{1}} D_{s_{2}} \ldots D_{s_{k}} \eta\right\|_{\infty}^{2^{k+1} p}\right),
\end{aligned}
$$

where $\eta \in \mathbb{L}^{\infty-}\left(\Omega, C([-\tau, 0], \mathbb{R}) ; \mathscr{F}_{\sigma}\right)$ are Malliavin smooth and such that

$$
\sup _{\sigma-\tau \leqslant s_{1}, \ldots, s_{k} \leqslant \sigma} E\left\|D_{s_{1}} D_{s_{2}} \ldots D_{s_{k}} \eta\right\|_{\infty}^{p}<\infty
$$

for every integer $k \geqslant 1$ and any $p \geqslant 1$. The positive constant $K$ is independent of $t \in[\sigma-\tau, T]$, $\sigma \in[0, T]$ and $\eta$.

Proof. This can be proved by following the same route as in the proofs of Lemmas 3.4 and 3.5 in $[\mathbf{1}]$.

Proof of Theorem 3.2. Let $t \in[\sigma, T]$ and

$$
\pi:=t_{-M}<t_{-M+1}<\ldots<t_{-1}<t_{0}=0<t_{1}<t_{2}<\ldots<t_{N-1}<t_{N}=T
$$

be a partition of $[-\tau, T]$ with equal size $|\pi|=(T+\tau) /(N+M)$. Without loss of generality, we assume that $\sigma=0$ and $t=t_{n} \in \pi$ for some $0 \leqslant n \leqslant N$. Suppose that $\eta \in H^{1, \infty}([-\tau, 0], \mathbb{R})$. 
Step 1. Using Lemma 4.2, we have

$$
\begin{aligned}
E & {\left[\phi\left(x\left(t_{n} ; 0, \eta\right)\right)\right]-E\left[\phi\left(y\left(t_{n} ; 0, \eta\right)\right)\right] } \\
& =E\left[\phi\left(y\left(t_{n} ; t_{n}, x_{t_{n}}(\cdot ; 0, \eta)\right)\right)\right]-E\left[\phi\left(y\left(t_{n} ; 0, \eta\right)\right)\right] \\
& =\sum_{i=1}^{n} E\left[\phi\left(y\left(t_{n} ; t_{i}, x_{t_{i}}(\cdot ; 0, \eta)\right)\right)-\phi\left(y\left(t_{n} ; t_{i-1}, x_{t_{i}}(\cdot ; 0, \eta)\right)\right)\right] \\
& =\sum_{i=1}^{n} E\left[\phi\left(y\left(t_{n} ; t_{i}, x_{t_{i}}\left(\cdot ; t_{i-1}, x_{t_{i-1}}(\cdot ; 0, \eta)\right)\right)\right)-\phi\left(y\left(t_{n} ; t_{i}, y_{t_{i}}\left(\cdot ; t_{i-1}, x_{t_{i-1}}(\cdot ; 0, \eta)\right)\right)\right)\right] .
\end{aligned}
$$

Step 2. Let $\psi:[0, \infty) \mapsto \mathbb{R}$ be a $C_{b}^{\infty}$ function ( $\psi$ and all of its partial derivatives are bounded) such that

$$
1_{\left[0, \frac{1}{8}\right]} \leqslant \psi \leqslant 1_{\left[0, \frac{1}{4}\right]} .
$$

Let us introduce

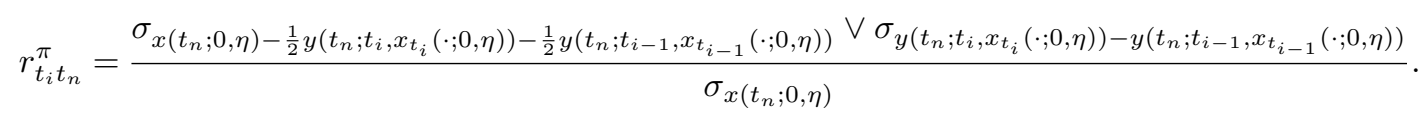

Now we split

$$
\sum_{i=1}^{n} E\left[\phi\left(y\left(t_{n} ; t_{i}, x_{t_{i}}\left(\cdot ; t_{i-1}, x_{t_{i-1}}(\cdot ; 0, \eta)\right)\right)\right)-\phi\left(y\left(t_{n} ; t_{i}, y_{t_{i}}\left(\cdot ; t_{i-1}, x_{t_{i-1}}(\cdot ; 0, \eta)\right)\right)\right)\right]
$$

into two parts:

$$
\begin{aligned}
\sum_{i=1}^{n} E & {\left[\phi\left(y\left(t_{n} ; t_{i}, x_{t_{i}}\left(\cdot ; t_{i-1}, x_{t_{i-1}}(\cdot ; 0, \eta)\right)\right)\right)-\phi\left(y\left(t_{n} ; t_{i}, y_{t_{i}}\left(\cdot ; t_{i-1}, x_{t_{i-1}}(\cdot ; 0, \eta)\right)\right)\right)\right] } \\
= & \sum_{i=1}^{n} E\left[\left(\phi\left(y\left(t_{n} ; t_{i}, x_{t_{i}}\left(\cdot ; t_{i-1}, x_{t_{i-1}}(\cdot ; 0, \eta)\right)\right)\right)\right.\right. \\
& \left.\left.-\phi\left(y\left(t_{n} ; t_{i}, y_{t_{i}}\left(\cdot ; t_{i-1}, x_{t_{i-1}}(\cdot ; 0, \eta)\right)\right)\right)\right)\left(1-\psi\left(r_{t_{i} t_{n}}^{\pi}\right)\right)\right] \\
& +\sum_{i=1}^{n} E\left[\left(\phi\left(y\left(t_{n} ; t_{i}, x_{t_{i}}\left(\cdot ; t_{i-1}, x_{t_{i-1}}(\cdot ; 0, \eta)\right)\right)\right)\right.\right. \\
& \left.\left.-\phi\left(y\left(t_{n} ; t_{i}, y_{t_{i}}\left(\cdot ; t_{i-1}, x_{t_{i-1}}(\cdot ; 0, \eta)\right)\right)\right)\right) \psi\left(r_{t_{i} t_{n}}^{\pi}\right)\right] .
\end{aligned}
$$

Step 3. First of all, we deal with the first part. Using the definition of $\psi$, for $p \geqslant 2$, we have

$$
\begin{aligned}
& P\left(\psi\left(r_{t_{i} t_{n}}^{\pi}\right) \neq 1\right) \leqslant P\left(\sigma_{x\left(t_{n} ; 0, \eta\right)}^{-1} \sigma_{x\left(t_{n} ; 0, \eta\right)-\frac{1}{2} y\left(t_{n} ; t_{i}, x_{t_{i}}(\cdot ; 0, \eta)\right)-\frac{1}{2} y\left(t_{n} ; t_{i-1}, x_{t_{i-1}}(\cdot ; 0, \eta)\right)}\right. \\
& \left.\vee \sigma_{y\left(t_{n} ; t_{i}, x_{t_{i}}(\cdot ; 0, \eta)\right)-y\left(t_{n} ; t_{i-1}, x_{t_{i-1}}(\cdot ; 0, \eta)\right)} \geqslant \frac{1}{8}\right) \\
& \leqslant 8^{p} E\left[\sigma _ { x ( t _ { n } ; 0 , \eta ) } ^ { - p } \left(\sigma_{x\left(t_{n} ; 0, \eta\right)-\frac{1}{2} y\left(t_{n} ; t_{i}, x_{t_{i}}(\cdot ; 0, \eta)\right)-\frac{1}{2} y\left(t_{n} ; t_{i-1}, x_{t_{i-1}}(\cdot ; 0, \eta)\right)}\right.\right.
\end{aligned}
$$

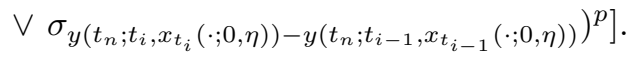

Using Lemma 4.1, we obtain

$$
\begin{aligned}
& E\left[\sigma_{x\left(t_{n} ; 0, \eta\right)-\frac{1}{2} y\left(t_{n} ; t_{i}, x_{t_{i}}(\cdot ; 0, \eta)\right)-\frac{1}{2} y\left(t_{n} ; t_{i-1}, x_{t_{i-1}}(\cdot ; 0, \eta)\right)}^{2 p}\right]^{1 / 2 p} \\
& \quad \leqslant E\left[\left(\sigma_{\frac{1}{2} x\left(t_{n} ; 0, \eta\right)-\frac{1}{2} y\left(t_{n} ; t_{i}, x_{t_{i}}(\cdot ; 0, \eta)\right)}^{1 / 2}+\sigma_{\frac{1}{2} x\left(t_{n} ; 0, \eta\right)-\frac{1}{2} y\left(t_{n} ; t_{i-1}, x_{t_{i-1}}(\cdot ; 0, \eta)\right)}^{1 / 2}\right)^{4 p}\right]^{1 / 2 p} \\
& \quad \leqslant\left\{E\left[\sigma_{\frac{1}{2} x\left(t_{n} ; 0, \eta\right)-\frac{1}{2} y\left(t_{n} ; t_{i}, x_{t_{i}}(\cdot ; 0, \eta)\right)}^{2 p}\right]^{1 / 4 p}+E\left[\sigma_{\frac{1}{2} x\left(t_{n} ; 0, \eta\right)-\frac{1}{2} y\left(t_{n} ; t_{i-1}, x_{t_{i-1}}(\cdot ; 0, \eta)\right)}^{2 p}\right]^{1 / 4 p}\right\}^{2} \\
& \quad \leqslant C|\pi|
\end{aligned}
$$


and

$$
\begin{aligned}
& E\left[\sigma_{y\left(t_{n} ; t_{i}, x_{t_{i}}(\cdot ; 0, \eta)\right)-y\left(t_{n} ; t_{i-1}, x_{t_{i-1}}(\cdot ; 0, \eta)\right)}^{2 p}\right]^{1 / 2 p} \\
& \quad \leqslant E\left[\left(\sigma_{y\left(t_{n} ; t_{i}, x_{t_{i}}(\cdot ; 0, \eta)\right)-x\left(t_{n} ; 0, \eta\right)}^{1 / 2}+\sigma_{x\left(t_{n} ; 0, \eta\right)-y\left(t_{n} ; t_{i-1}, x_{t_{i-1}}(\cdot ; 0, \eta)\right)}^{1 / 2}\right)^{4 p}\right]^{1 / 2 p} \\
& \quad \leqslant\left\{E\left[\sigma_{y\left(t_{n} ; t_{i}, x_{t_{i}}(\cdot ; 0, \eta)\right)-x\left(t_{n} ; 0, \eta\right)}^{2 p}\right]^{1 / 4 p}+E\left[\sigma_{x\left(t_{n} ; 0, \eta\right)-y\left(t_{n} ; t_{i-1}, x_{t_{i-1}}(\cdot ; 0, \eta)\right)}^{2 p}\right]^{1 / 4 p}\right\}^{2} \\
& \quad \leqslant C|\pi| .
\end{aligned}
$$

Therefore, we have

$$
P\left(\psi\left(r_{t_{i} t_{n}}^{\pi}\right) \neq 1\right) \leqslant C|\pi|^{p}
$$

Consequently, from the boundedness of $\phi$, we obtain

$$
\begin{aligned}
& \mid E\left[\left(\phi\left(y\left(t_{n} ; t_{i}, x_{t_{i}}\left(\cdot ; t_{i-1}, x_{t_{i-1}}(\cdot ; 0, \eta)\right)\right)\right)\right.\right. \\
& \left.\quad-\phi\left(y\left(t_{n} ; t_{i}, y_{t_{i}}\left(\cdot ; t_{i-1}, x_{t_{i-1}}(\cdot ; 0, \eta)\right)\right)\right)\left(1-\psi\left(r_{t_{i} t_{n}}^{\pi}\right)\right)\right] \mid \\
& \quad \leqslant C|\pi|^{p} .
\end{aligned}
$$

Since $|\pi|=(T+\tau) /(N+M)$ and $0 \leqslant n \leqslant N$, then we obtain

$$
\begin{aligned}
& \mid \sum_{i=1}^{n} E\left[\phi\left(y\left(t_{n} ; t_{i}, x_{t_{i}}\left(\cdot ; t_{i-1}, x_{t_{i-1}}(\cdot ; 0, \eta)\right)\right)\right)\right. \\
& \left.\quad-\phi\left(y\left(t_{n} ; t_{i}, y_{t_{i}}\left(\cdot ; t_{i-1}, x_{t_{i-1}}(\cdot ; 0, \eta)\right)\right)\right)\left(1-\psi\left(r_{t_{i} t_{n}}^{\pi}\right)\right)\right] \mid \\
& \quad \leqslant C|\pi|^{p-1},
\end{aligned}
$$

and, hence, we complete the proof of the first part.

Step 4. It remains to prove that the second part of (24) is of order $|\pi|$. Let $\left\{\phi_{h}\right\}_{h=1}^{\infty}$ be a sequence of $C^{1}$ functions such that $\left\|\phi_{h}\right\|_{\infty} \leqslant\|\phi\|_{\infty}$ and $\left\{\phi_{h}\right\}_{h=1}^{\infty}$ converges almost every $d x$ to $\phi$ as $h$ tends to infinity. Now on the set $\left\{\psi\left(r_{t_{i} t_{n}}^{\pi}\right) \neq 0\right\}$, we have

$$
\operatorname{det}\left(\sigma_{y\left(t_{n} ; t_{i}, x_{t_{i}}\left(\cdot ; t_{i-1}, x_{t_{i-1}}(\cdot ; 0, \eta)\right)\right)}\right)>0 \quad \text { and } \operatorname{det}\left(\sigma_{y\left(t_{n} ; t_{i}, y_{t_{i}}\left(\cdot ; t_{i-1}, x_{t_{i-1}}(\cdot ; 0, \eta)\right)\right)}\right)>0 .
$$

To prove this result, we proceed as follows. From Lemma 4.2, it suffices to show $\operatorname{det}\left(\sigma_{y\left(t_{n} ; t_{i}, x_{t_{i}}(\cdot ; 0, \eta)\right)}\right)>0$ and $\operatorname{det}\left(\sigma_{y\left(t_{n} ; t_{i-1}, x_{t_{i-1}}(\cdot ; 0, \eta)\right)}\right)>0$ on the set $\left\{\psi\left(r_{t_{i} t_{n}}^{\pi}\right) \neq 0\right\}$. Since

$$
\begin{aligned}
& \sigma_{\lambda y\left(t_{n} ; t_{i}, x_{t_{i}}(\cdot ; 0, \eta)\right)+(1-\lambda) y\left(t_{n} ; t_{i-1}, x_{t_{i-1}}(\cdot ; 0, \eta)\right)} \\
& \geqslant \frac{1}{2} \sigma_{\frac{1}{2} y\left(t_{n} ; t_{i}, x_{t_{i}}(\cdot ; 0, \eta)\right)+\frac{1}{2} y\left(t_{n} ; t_{i-1}, x_{t_{i-1}}(\cdot ; 0, \eta)\right)} \\
& \quad-\left(\lambda-\frac{1}{2}\right)^{2} \sigma_{y\left(t_{n} ; t_{i}, x_{t_{i}}(\cdot ; 0, \eta)\right)-y\left(t_{n} ; t_{i-1}, x_{t_{i-1}}(\cdot ; 0, \eta)\right)} \\
& \geqslant \frac{1}{4} \sigma_{x\left(t_{n} ; 0, \eta\right)}-\frac{1}{2} \sigma_{x\left(t_{n} ; 0, \eta\right)-\frac{1}{2} y\left(t_{n} ; t_{i}, x_{t_{i}}(\cdot ; 0, \eta)\right)-\frac{1}{2} y\left(t_{n} ; t_{i-1}, x_{t_{i-1}}(\cdot ; 0, \eta)\right)} \\
&-\left(\lambda-\frac{1}{2}\right)^{2} \sigma_{y\left(t_{n} ; t_{i}, x_{t_{i}}(\cdot ; 0, \eta)\right)-y\left(t_{n} ; t_{i-1}, x_{t_{i-1}}(\cdot ; 0, \eta)\right)}
\end{aligned}
$$

and

$$
\begin{aligned}
\left\{\psi\left(r_{t_{i} t_{n}}^{\pi}\right) \neq 0\right\} & \subset\left\{\sigma_{x\left(t_{n} ; 0, \eta\right)-\frac{1}{2} y\left(t_{n} ; t_{i}, x_{t_{i}}(\cdot ; 0, \eta)\right)-\frac{1}{2} y\left(t_{n} ; t_{i-1}, x_{t_{i-1}}(\cdot ; 0, \eta)\right)}\right. \\
& \left.\vee \sigma_{y\left(t_{n} ; t_{i}, x_{t_{i}}(\cdot ; 0, \eta)\right)-y\left(t_{n} ; t_{i-1}, x_{t_{i-1}}(\cdot ; 0, \eta)\right)} \leqslant \frac{1}{4} \sigma_{x\left(t_{n} ; 0, \eta\right)}\right\},
\end{aligned}
$$

then for all $0 \leqslant \lambda \leqslant 1$, we obtain

$$
\left\{\psi\left(r_{t_{i} t_{n}}^{\pi}\right) \neq 0\right\} \subset\left\{\sigma_{\lambda y\left(t_{n} ; t_{i}, x_{t_{i}}(\cdot ; 0, \eta)\right)+(1-\lambda) y\left(t_{n} ; t_{i-1}, x_{t_{i-1}}(\cdot ; 0, \eta)\right)} \geqslant \frac{1}{16} \sigma_{x\left(t_{n} ; 0, \eta\right)}\right\},
$$

and this gives $\operatorname{det}\left(\sigma_{y\left(t_{n} ; t_{i}, x_{t_{i}}(\cdot ; 0, \eta)\right)}\right)>0$ and $\operatorname{det}\left(\sigma_{y\left(t_{n} ; t_{i-1}, x_{t_{i-1}}(\cdot ; 0, \eta)\right)}\right)>0$ on the set $\left\{\psi\left(r_{t_{i} t_{n}}^{\pi}\right) \neq\right.$ $0\}$. Then from [14, Theorem 2.13] (see [5] in higher dimension), $y\left(t_{n} ; t_{i}, y_{t_{i}}\left(\cdot ; t_{i-1}, x_{t_{i-1}}(\cdot ; 0, \eta)\right)\right.$ ) 
and $y\left(t_{n} ; t_{i}, x_{t_{i}}\left(\cdot ; t_{i-1}, x_{t_{i-1}}(\cdot ; 0, \eta)\right)\right)$ have absolutely continuous law conditioned by the set $\left\{\psi\left(r_{t_{i} t_{n}}^{\pi}\right) \neq 0\right\}$. Thus, we have

$$
\begin{aligned}
& E\left[\phi_{h}\left(y\left(t_{n} ; t_{i}, x_{t_{i}}\left(\cdot ; t_{i-1}, x_{t_{i-1}}(\cdot ; 0, \eta)\right)\right)\right) \psi\left(r_{t_{i} t_{n}}^{\pi}\right)\right] \\
& \quad \rightarrow E\left[\phi\left(y\left(t_{n} ; t_{i}, x_{t_{i}}\left(\cdot ; t_{i-1}, x_{t_{i-1}}(\cdot ; 0, \eta)\right)\right)\right) \psi\left(r_{t_{i} t_{n}}^{\pi}\right)\right]
\end{aligned}
$$

and

$$
\begin{aligned}
& E\left[\phi_{h}\left(y\left(t_{n} ; t_{i}, y_{t_{i}}\left(\cdot ; t_{i-1}, x_{t_{i-1}}(\cdot ; 0, \eta)\right)\right)\right) \psi\left(r_{t_{i} t_{n}}^{\pi}\right)\right] \\
& \quad \rightarrow E\left[\phi\left(y\left(t_{n} ; t_{i}, y_{t_{i}}\left(\cdot ; t_{i-1}, x_{t_{i-1}}(\cdot ; 0, \eta)\right)\right)\right) \psi\left(r_{t_{i} t_{n}}^{\pi}\right)\right]
\end{aligned}
$$

as $h$ tends to infinity. Therefore, it now suffices to prove that

$$
\begin{aligned}
& \mid \sum_{i=1}^{n} E\left[\left(\phi_{h}\left(y\left(t_{n} ; t_{i}, x_{t_{i}}\left(\cdot ; t_{i-1}, x_{t_{i-1}}(\cdot ; 0, \eta)\right)\right)\right)\right.\right. \\
& \left.\left.\quad-\phi_{h}\left(y\left(t_{n} ; t_{i}, y_{t_{i}}\left(\cdot ; t_{i-1}, x_{t_{i-1}}(\cdot ; 0, \eta)\right)\right)\right)\right) \psi\left(r_{t_{i} t_{n}}^{\pi}\right)\right] \mid
\end{aligned}
$$

is of order $|\pi|$.

Step 5. Applying the mean value theorem and Lemma 4.3, we have

$$
\begin{aligned}
E[ & \left(\phi_{h}\left(y\left(t_{n} ; t_{i}, x_{t_{i}}\left(\cdot ; t_{i-1}, x_{t_{i-1}}(\cdot ; 0, \eta)\right)\right)\right)-\phi_{h}\left(y\left(t_{n} ; t_{i}, y_{t_{i}}\left(\cdot ; t_{i-1}, x_{t_{i-1}}(\cdot ; 0, \eta)\right)\right)\right) \psi\left(r_{t_{i} t_{n}}^{\pi}\right)\right] \\
= & \int_{0}^{1} E\left[\nabla\left(\phi_{h} \circ y\right)\left(t_{n} ; t_{i}, \lambda x_{t_{i}}\left(\cdot ; t_{i-1}, x_{t_{i-1}}(\cdot ; 0, \eta)\right)+(1-\lambda) y_{t_{i}}\left(\cdot ; t_{i-1}, x_{t_{i-1}}(\cdot ; 0, \eta)\right)\right)\right. \\
& \left.\times\left(x_{t_{i}}\left(\cdot ; t_{i-1}, x_{t_{i-1}}(\cdot ; 0, \eta)\right)-y_{t_{i}}\left(\cdot ; t_{i-1}, x_{t_{i-1}}(\cdot ; 0, \eta)\right)\right) \psi\left(r_{t_{i} t_{n}}^{\pi}\right)\right] d \lambda \\
= & \int_{0}^{1} \sum_{m=1}^{l} E\left[\nabla \phi_{h}\left(y\left(t_{n} ; t_{i}, \lambda x_{t_{i}}\left(\cdot ; t_{i-1}, x_{t_{i-1}}(\cdot ; 0, \eta)\right)+(1-\lambda) y_{t_{i}}\left(\cdot ; t_{i-1}, x_{t_{i-1}}(\cdot ; 0, \eta)\right)\right)\right)\right. \\
& \times \partial_{m} F\left(t, W(t), W\left(t_{1}\right), \ldots, W\left(t_{k}\right), s_{1}, \ldots, s_{n}, \ldots, \lambda x\left(t_{i}+\mu_{m}\right)+(1-\lambda) y\left(t_{i}+\mu_{m}\right), \ldots\right) \\
& \left.\times\left(x_{t_{i}}\left(\mu_{m} ; t_{i-1}, x_{t_{i-1}}(\cdot ; 0, \eta)\right)-y_{t_{i}}\left(\mu_{m} ; t_{i-1}, x_{t_{i-1}}(\cdot ; 0, \eta)\right)\right) \psi\left(r_{t_{i} t_{n}}^{\pi}\right)\right] d \lambda .
\end{aligned}
$$

Let

$$
F_{i}=y\left(t_{n} ; t_{i}, \lambda x_{t_{i}}\left(\cdot ; t_{i-1}, x_{t_{i-1}}(\cdot ; 0, \eta)\right)+(1-\lambda) y_{t_{i}}\left(\cdot ; t_{i-1}, x_{t_{i-1}}(\cdot ; 0, \eta)\right)\right)
$$

and

$$
\begin{aligned}
G_{i m}= & \partial_{m} F\left(t, W(t), W\left(t_{1}\right), \ldots, W\left(t_{k}\right), s_{1}, \ldots, s_{n}, \ldots, \lambda x\left(t_{i}+\mu_{m}\right)+(1-\lambda) y\left(t_{i}+\mu_{m}\right), \ldots\right) \\
& \times\left(x_{t_{i}}\left(\cdot ; t_{i-1}, x_{t_{i-1}}(\cdot ; 0, \eta)\right)-y_{t_{i}}\left(\cdot ; t_{i-1}, x_{t_{i-1}}(\cdot ; 0, \eta)\right)\right) \psi\left(r_{t_{i} t_{n}}^{\pi}\right) .
\end{aligned}
$$

By Lemma 4.2, we know that

$$
F_{i}=\lambda y\left(t_{n} ; t_{i}, x_{t_{i}}(\cdot ; 0, \eta)\right)+(1-\lambda) y\left(t_{n} ; t_{i-1}, x_{t_{i-1}}(\cdot ; 0, \eta)\right) .
$$

Therefore from (25), we obtain that $F_{i}$ is non-degenerate on the set $\left\{\psi\left(r_{t_{i} t_{n}}^{\pi}\right) \neq 0\right\}$. Thus, applying the local integration by parts formula which is stated in Proposition 2.2, we obtain

$$
E\left[\nabla \phi_{h}\left(F_{i}\right) G_{i m}\right]=E\left[U\left(F_{i}\right) H_{3}\left(F_{i}, G_{i m}\right)\right],
$$

where $U(x)=\int_{0}^{x} \int_{0}^{y} \phi_{h}(z) d z d y$ and $H_{3}$ is defined in Proposition 2.2. It is obvious that $U$ is in $C^{3}$ and $U^{\prime \prime}=\phi_{h}$.

Step 6. Observe that $H_{3}\left(F_{i}, G_{i m}\right)$ can be rewritten as follows:

$$
H_{3}\left(F_{i}, G_{i m}\right)=\sum_{k=0}^{3}\left\langle\Phi_{k}, D^{k} G_{i m}\right\rangle,
$$


where

$$
\Phi_{k} \in \mathbb{D}_{\infty}^{\infty-}\left(H^{\otimes k}\right), \quad k=0,1,2,3,
$$

which are obtained as polynomials in $\gamma_{F_{i}}, F_{i}$ and their derivatives (cf. [7]). Thus, our problem can be reformulated to prove

$$
\left|\sum_{k=0}^{3} \sum_{i=1}^{n} \sum_{m=1}^{l} \int_{0}^{1} E\left[\left\langle U\left(F_{i}\right) \Phi_{k}, D^{k} G_{i m}\right\rangle\right] d \lambda\right|
$$

is of order $|\pi|$. To prove this result, we just have to show each of term

$$
\mid \sum_{i=1}^{n} \sum_{m=1}^{l} \int_{0}^{1} E\left[U\left(F_{i}\right)\left\langle\Phi_{k}, D^{k} G_{i m}\right\rangle\right] d \lambda, \quad k=0,1,2,3
$$

is of order $|\pi|$. For the case $k=0$, using a similar argument as in [1, Theorem 3.1] we can obtain the desired result. For the remaining cases, we only consider the case $k=1$, and the other cases can be estimated through the very similar procedure. Since $H=L^{2}([0, T], \mathbb{R})$, then we have

$$
\begin{aligned}
& \sum_{i=1}^{n} \sum_{m=1}^{l} \int_{0}^{1} E\left[U\left(F_{i}\right)\left\langle\Phi_{1}, D G_{i m}\right\rangle_{H}\right] d \lambda \\
& =\sum_{i=1}^{n} \sum_{m=1}^{l} \int_{0}^{1} E\left[U\left(F_{i}\right) \int_{0}^{T} \Phi_{1}(r) D_{r} G_{i m} d r\right] d \lambda \\
& =\sum_{i=1}^{n} \sum_{m=1}^{l} \int_{0}^{1} \int_{0}^{T} E\left[U\left(F_{i}\right) \Phi_{1}(r) D_{r} G_{i m}\right] d r d \lambda .
\end{aligned}
$$

Substituting $G_{i m}$ in (26) into $U\left(F_{i}\right) \Phi_{1}(r) D_{r} G_{i m}$, we have

$$
\begin{aligned}
U\left(F_{i}\right) \Phi_{1}(r) D_{r} G_{i m}= & U\left(F_{i}\right) \Phi_{1}(r) D_{r}\left(\partial _ { m } F \left(t, W(t), W\left(t_{1}\right), \ldots, W\left(t_{k}\right),\right.\right. \\
& \left.s_{1}, \ldots, s_{n}, \ldots, \lambda x\left(t_{i}+\mu_{m}\right)+(1-\lambda) y\left(t_{i}+\mu_{m}\right), \ldots\right) \\
& \left.\times\left(x_{t_{i}}\left(\mu_{m} ; t_{i-1}, x_{t_{i-1}}(\cdot ; 0, \eta)\right)-y_{t_{i}}\left(\mu_{m} ; t_{i-1}, x_{t_{i-1}}(\cdot ; 0, \eta)\right)\right) \psi\left(r_{t_{i} t_{n}}^{\pi}\right)\right) \\
= & U\left(F_{i}\right) \Phi_{1}(r) D_{r}\left(\partial _ { m } F \left(t, W(t), W\left(t_{1}\right), \ldots, W\left(t_{k}\right), s_{1}, \ldots, s_{n}, \ldots,\right.\right. \\
& \left.\left.\lambda x\left(t_{i}+\mu_{m}\right)+(1-\lambda) y\left(t_{i}+\mu_{m}\right), \ldots\right)\right) \\
& \times\left(x_{t_{i}}\left(\mu_{m} ; t_{i-1}, x_{t_{i-1}}(\cdot ; 0, \eta)\right)-y_{t_{i}}\left(\mu_{m} ; t_{i-1}, x_{t_{i-1}}(\cdot ; 0, \eta)\right)\right) \psi\left(r_{t_{i} t_{n}}^{\pi}\right) \\
& +U\left(F_{i}\right) \Phi_{1}(r) \partial_{m} F\left(t, W(t), W\left(t_{1}\right), \ldots, W\left(t_{k}\right), s_{1}, \ldots, s_{n}, \ldots,\right. \\
& \left.\lambda x\left(t_{i}+\mu_{m}\right)+(1-\lambda) y\left(t_{i}+\mu_{m}\right), \ldots\right) \\
& \times\left(x_{t_{i}}\left(\mu_{m} ; t_{i-1}, x_{t_{i-1}}(\cdot ; 0, \eta)\right)-y_{t_{i}}\left(\mu_{m} ; t_{i-1}, x_{t_{i-1}}(\cdot ; 0, \eta)\right)\right) D_{r} \psi\left(r_{t_{i} t_{n}}^{\pi}\right) \\
& +U\left(F_{i}\right) \Phi_{1}(r) \partial_{m} F\left(t, W(t), W\left(t_{1}\right), \ldots, W\left(t_{k}\right), s_{1}, \ldots, s_{n}, \ldots,\right. \\
& \left.\lambda x\left(t_{i}+\mu_{m}\right)+(1-\lambda) y\left(t_{i}+\mu_{m}\right), \ldots\right) \\
& \times D_{r}\left(\left(x_{t_{i}}\left(\mu_{m} ; t_{i-1}, x_{t_{i-1}}(\cdot ; 0, \eta)\right)-y_{t_{i}}\left(\mu_{m} ; t_{i-1}, x_{t_{i-1}}(\cdot ; 0, \eta)\right)\right)\right) \psi\left(r_{t_{i} t_{n}}^{\pi}\right) \\
:= & B_{i m 1}+B_{i m 2}+B_{i m 3} .
\end{aligned}
$$

Thus, our main objection is to show

$$
\left|\sum_{i=1}^{n} \sum_{m=1}^{l} \int_{0}^{1} \int_{0}^{T} E\left[B_{i m k}\right] d r d \lambda\right|, \quad k=1,2,3
$$

is of order $|\pi|$. 
Step 7. For the first two terms, we have the following estimate:

$$
\left|\sum_{i=1}^{n} \sum_{m=1}^{l} \int_{0}^{1} \int_{0}^{T} E\left[B_{i m k}\right] d r d \lambda\right| \leqslant C\left(1+\|\eta\|_{1, \infty}^{q}\right)|\pi|, \quad k=1,2 .
$$

This is done using the same arguments as in the proof of [1, Theorem 3.1]. Note that we must use Lemma 4.5 in the proof of the above estimates.

Step 8. Next we mainly deal with the third term. Using Lemma 4.4, we obtain

$$
\begin{aligned}
\sum_{i=1}^{n} & \sum_{m=1}^{l} \int_{0}^{1} \int_{0}^{T} E\left[B_{i m 3}\right] \\
= & \sum_{i=1}^{n} \sum_{m=1}^{l} \int_{0}^{1} \int_{0}^{T} E\left[U ( F _ { i } ) \Phi _ { 1 } ( r ) \partial _ { m } F \left(t, W(t), W\left(t_{1}\right), \ldots, W\left(t_{k}\right),\right.\right. \\
& \left.s_{1}, \ldots, s_{n}, \ldots, \lambda x\left(t_{i}+\mu_{m}\right)+(1-\lambda) y\left(t_{i}+\mu_{m}\right), \ldots\right) \\
& \left.\times D_{r}\left(\left(x_{t_{i}}\left(\mu_{m} ; t_{i-1}, x_{t_{i-1}}(\cdot ; 0, \eta)\right)-y_{t_{i}}\left(\mu_{m} ; t_{i-1}, x_{t_{i-1}}(\cdot ; 0, \eta)\right)\right) \psi\left(r_{t_{i} t_{n}}^{\pi}\right)\right)\right] d r d \lambda \\
= & \sum_{j=1}^{19} \sum_{i=1}^{n} \sum_{m=1}^{l} \int_{0}^{1} \int_{0}^{T} E\left[U ( F _ { i } ) \Phi _ { 1 } ( r ) \partial _ { m } F \left(t, W(t), W\left(t_{1}\right), \ldots, W\left(t_{k}\right), s_{1}, \ldots, s_{n}, \ldots,\right.\right. \\
& \left.\left.\lambda x\left(t_{i}+\mu_{m}\right)+(1-\lambda) y\left(t_{i}+\mu_{m}\right), \ldots\right) \Xi_{j}\left(\mu_{m}\right) \psi\left(r_{t_{i} t_{n}}^{\pi}\right)\right] d r d \lambda \\
:= & \sum_{j=1}^{19} \Pi_{j} .
\end{aligned}
$$

For the cases $j=1,2,3,4,5,6,7,8,9,10$, we obtain $\left|\Pi_{j}\right|$ is of order $|\pi|$. This result can be found using the same technique as in the proof of $[\mathbf{1}$, Theorem 3.1]. For the case $j=11,12,13,14,15$, it suffices to consider the case $j=13$, and the other cases can be estimated similarly. Substituting $\Xi_{13}$ in (14) into $\Pi_{13}$, we have

$$
\begin{aligned}
\Pi_{13}= & \sum_{i=1}^{n} \sum_{m=1}^{l} \int_{0}^{1} \int_{0}^{T} E\left[U ( F _ { i } ) \Phi _ { 1 } ( r ) \partial _ { m } F \left(t, W(t), W\left(t_{1}\right), \ldots, W\left(t_{k}\right), s_{1}, \ldots, s_{n}, \ldots,\right.\right. \\
& \left.\left.\lambda x\left(t_{i}+\mu_{m}\right)+(1-\lambda) y\left(t_{i}+\mu_{m}\right), \ldots\right) \Xi_{13}\left(\mu_{m}, r\right) \psi\left(r_{t_{i} t_{n}}^{\pi}\right)\right] d r d \lambda \\
= & \sum_{i=1}^{n} \sum_{m=1}^{l} \int_{0}^{1} \int_{0}^{T} E\left[U ( F _ { i } ) \Phi _ { 1 } ( r ) \partial _ { m } F \left(t, W(t), W\left(t_{1}\right), \ldots, W\left(t_{k}\right), s_{1}, \ldots, s_{n}, \ldots,\right.\right. \\
& \left.\lambda x\left(t_{i}+\mu_{m}\right)+(1-\lambda) y\left(t_{i}+\mu_{m}\right), \ldots\right) \\
& \times \int_{[r]}^{r} \frac{\partial^{2} g}{\partial x_{1} \partial x_{2}}(x(v-\tau), x(v)) g(x(v-2 \tau), x(v-\tau)) \\
& \left.\times 1_{[\tau, \infty)}(v) D_{v-\tau} x(v) d v 1_{\left(t_{i-1},\left(t_{i}+s\right) \vee t_{i-1}\right]}(r) \psi\left(r_{t_{i} t_{n}}^{\pi}\right)\right] d r d \lambda \\
= & \sum_{i=1}^{n} \sum_{m=1}^{l} \int_{0}^{1} \int_{t_{i-1}}^{\left(t_{i}+\mu_{m}\right) \vee t_{i-1}} E\left[U ( F _ { i } ) \Phi _ { 1 } ( r ) \partial _ { m } F \left(t, W(t), W\left(t_{1}\right), \ldots, W\left(t_{k}\right),\right.\right. \\
& \left.s_{1}, \ldots, s_{n}, \ldots, \lambda x\left(t_{i}+\mu_{m}\right)+(1-\lambda) y\left(t_{i}+\mu_{m}\right), \ldots\right) \\
& \times \int_{[r]}^{r} \frac{\partial^{2} g}{\partial x_{1} \partial x_{2}}(x(v-\tau), x(v)) g(x(v-2 \tau), x(v-\tau)) \\
& \left.\times 1_{[\tau, \infty)}(v) D_{v-\tau} x(v) d v \psi\left(r_{t_{i} t_{n}}^{\pi}\right)\right] d r d \lambda .
\end{aligned}
$$

Therefore, we observe that the term $\Pi_{13}$ has a similar form to $\Pi_{i}, i=1, \ldots, 10$. Thus, applying the same procedure as used in $\left[\mathbf{1}\right.$, Theorem 3.1], we obtain $\left|\Pi_{13}\right|$ is of order $|\pi|$. It remains to 
estimate the last four terms $\Pi_{j}, j=16,17,18,19$. For the case $j=16$, we proceed as follows:

$$
\begin{aligned}
& \Pi_{16}= \sum_{i=1}^{n} \sum_{m=1}^{l} \int_{0}^{1} \int_{0}^{T} E\left[U ( F _ { i } ) \Phi _ { 1 } ( r ) \partial _ { m } F \left(t, W(t), W\left(t_{1}\right), \ldots, W\left(t_{k}\right), s_{1}, \ldots, s_{n}, \ldots,\right.\right. \\
&\left.\left.\lambda x\left(t_{i}+\mu_{m}\right)+(1-\lambda) y\left(t_{i}+\mu_{m}\right), \ldots\right) \Xi_{16}\left(\mu_{m}, r\right)\right] d r d \lambda \\
&= \sum_{i=1}^{n} \sum_{m=1}^{l} \int_{0}^{1} \int_{0}^{T} E\left[U ( F _ { i } ) \Phi _ { 1 } ( r ) \partial _ { m } F \left(t, W(t), W\left(t_{1}\right), \ldots, W\left(t_{k}\right), s_{1}, \ldots, s_{n}, \ldots,\right.\right. \\
&\left.\lambda x\left(t_{i}+\mu_{m}\right)+(1-\lambda) y\left(t_{i}+\mu_{m}\right), \ldots\right) \\
& \times \int_{t_{i-1}}^{\left(t_{i}+\mu_{m}\right) \vee t_{i-1}} g(x(r-\tau), x(r)) g(x(r-\tau), x(r)) \\
&\left.\times 1_{([u]-\tau, u-\tau]}(r) 1_{\tau<[u]} \frac{\partial}{\partial x_{1}} g(x([u]-\tau), x([u])) d W(u)\right] d r d \lambda \\
&+\sum_{i=1}^{n} \sum_{m=1}^{l} \int_{0}^{1} \int_{0}^{T} E\left[U ( F _ { i } ) \Phi ( r ) \partial _ { m } F \left(t, W(t), W\left(t_{1}\right), \ldots, W\left(t_{k}\right), s_{1}, \ldots, s_{n}, \ldots,\right.\right. \\
&\left.\lambda x\left(t_{i}+\mu_{m}\right)+(1-\lambda) y\left(t_{i}+\mu_{m}\right), \ldots\right) \\
& \times \int_{t_{i-1}}^{\left(t_{i}+\mu_{m}\right) \vee t_{i-1}} g(x(r-\tau), x(r)) 1_{(0, u-\tau]}(r) 1_{[u] \leqslant \tau<u} \\
&\left.\times \frac{\partial}{\partial x_{1}} g(x([u]-\tau), x([u])) d W(u)\right] d r d \lambda \\
&+\sum_{i=1}^{n} \sum_{m=1}^{l} \int_{0}^{1} \int_{0}^{T} E\left[U ( F _ { i } ) \Phi ( r ) \partial _ { m } F \left(t, W(t), W\left(t_{1}\right), \ldots, W\left(t_{k}\right), s_{1}, \ldots, s_{n}, \ldots,\right.\right. \\
&\left.\lambda x\left(t_{i}+\mu_{m}\right)+(1-\lambda) y\left(t_{i}+\mu_{m}\right), \ldots\right) \int_{t_{i-1}}^{\left(t_{i}+\mu_{m}\right) \vee t_{i-1}} g(x(r-\tau), x(r)) 1_{([u], u]}(r) \\
&\left.\times \frac{\partial}{\partial x_{2}} g(x([u]-\tau), x([u])) d W(u)\right] d r d \lambda \\
& R_{1}+R_{2}+R_{3} .
\end{aligned}
$$

We only deal with the term $R_{2}$, and the estimate of the other two terms $R_{1}$ and $R_{3}$ is similar and we omit it. Using the definition of the Skorohod integral as an adjoint of the Malliavin derivative, we can rewrite the term $R_{2}$ into the following forms:

$$
\begin{aligned}
R_{2}= & \sum_{i=1}^{n} \sum_{m=1}^{l} \int_{0}^{1} E\left[U ( F _ { i } ) \Phi _ { 1 } ( r ) \partial _ { m } F \left(t, W(t), W\left(t_{1}\right), \ldots, W\left(t_{k}\right), s_{1}, \ldots, s_{n}, \ldots,\right.\right. \\
& \left.\lambda x\left(t_{i}+\mu_{m}\right)+(1-\lambda) y\left(t_{i}+\mu_{m}\right), \ldots\right) \\
& \times \int_{t_{i-1}}^{\left(t_{i}+\mu_{m}\right) \vee t_{i-1}} \int_{0}^{T} g(x(r-\tau), x(r)) 1_{(0, u-\tau]}(r) 1_{[u] \leqslant \tau<u} \\
& \left.\times \frac{\partial}{\partial x_{1}} g(x([u]-\tau), x([u])) d r d W(u)\right] d \lambda \\
= & \sum_{i=1}^{n} \sum_{m=1}^{l} \int_{0}^{1} \int_{t_{i-1}}^{\left(t_{i}+\mu_{m}\right) \vee t_{i-1}} E\left[D _ { u } ( U ( F _ { i } ) \Phi _ { 1 } ( r ) ) \partial _ { m } F \left(t, W(t), W\left(t_{1}\right), \ldots, W\left(t_{k}\right),\right.\right.
\end{aligned}
$$




$$
\begin{aligned}
& \left.s_{1}, \ldots, s_{n}, \ldots, \lambda x\left(t_{i}+\mu_{m}\right)+(1-\lambda) y\left(t_{i}+\mu_{m}\right), \ldots\right) \int_{0}^{u-\tau} g(x(r-\tau), x(r)) 1_{[u] \leqslant \tau<u} \\
& \left.\times \frac{\partial}{\partial x_{1}} g(x([u]-\tau), x([u])) d r\right] d u d \lambda \\
& +\sum_{i=1}^{n} \sum_{m=1}^{l} \int_{0}^{1} \int_{t_{i-1}}^{\left(t_{i}+\mu_{m}\right) \vee t_{i-1}} E\left[U ( F _ { i } ) \Phi _ { 1 } ( r ) D _ { u } \partial _ { m } F \left(t, W(t), W\left(t_{1}\right), \ldots, W\left(t_{k}\right),\right.\right. \\
& \left.s_{1}, \ldots, s_{n}, \ldots, \lambda x\left(t_{i}+\mu_{m}\right)+(1-\lambda) y\left(t_{i}+\mu_{m}\right), \ldots\right) \\
& \left.\times \int_{0}^{u-\tau} g(x(r-\tau), x(r)) 1_{[u] \leqslant \tau<u} \frac{\partial}{\partial x_{1}} g(x([u]-\tau), x([u])) d r\right] d u d \lambda .
\end{aligned}
$$

Since under the condition $[u] \leqslant \tau<u$, we have $u-\tau<u-[u]$, then applying a similar procedure to that used in $[\mathbf{1}]$, we can obtain that $\left|R_{2}\right|$ is of order $|\pi|$. Similarly, for the remaining terms $\Pi_{17}, \Pi_{18}$ and $\Pi_{19}$, we proceed in the same way. Through the above steps, we have shown there is a positive constant $K$ and a positive integer $q$ such that

$$
|E[\phi(x(t ; \sigma, \eta))]-E[\phi(y(t ; \sigma, \eta))]| \leqslant K\left(1+\|\eta\|_{1, \infty}^{q}\right)|\pi|
$$

for all $t \in[\sigma-\tau, T], \sigma \in[0, T]$ and all $\eta \in H^{1, \infty}([-\tau, 0], \mathbb{R})$

Step 9. Using [12, Theorem 3.1], we have

$$
\left|E[\phi(x(t ; \sigma, \eta))]-E\left[\phi\left(x\left(t ; \sigma, \eta^{\pi}\right)\right)\right]\right| \leqslant K\left\|\eta-\eta^{\pi}\right\|_{C} .
$$

Also we have that the following two elementary estimates hold

$$
\left\|\eta-\eta^{\pi}\right\|_{C} \leqslant 2|\nabla \eta|_{\infty}|\pi|, \quad\left\|\eta^{\pi}\right\|_{1, \infty} \leqslant\|\eta\|_{1, \infty}
$$

for all $\eta \in H^{1, \infty}([-\tau, 0], \mathbb{R})$. Combined with the above estimates, the proof of Theorem 3.2 is now complete.

\section{Higher dimension version of Theorem 3.2}

In this section, we extend Theorem 3.2 to higher dimension. More precisely, we study the case of $\mathbb{R}^{d}$-valued SFDEs driven by multidimensional Brownian motion and having several discrete delays in the drift and diffusion coefficients, as well as (smooth) quasi-tame dependence on the history of the solution in all coefficients.

The following notation is taken from [1]. Let $W(t):=\left(W_{1}(t), W_{2}(t), \ldots, W_{m}(t)\right), t \geqslant 0$, be $m$-dimensional standard Brownian motion on a filtered probability space $\left(\Omega, \mathscr{F},\left(\mathscr{F}_{t}\right)_{t \geqslant 0}, P\right)$. Consider a finite number of delays $\left\{\tau_{1}^{i}: 1 \leqslant i \leqslant k_{1}\right\}, \quad\left\{\tau_{2}^{j, l}: 1 \leqslant j \leqslant k_{2, l}, 1 \leqslant l \leqslant m\right\}$, with maximum delay $\tau:=\max \left\{\tau_{1}^{i}, \tau_{2}^{j, l}: 1 \leqslant i \leqslant k_{1}, 1 \leqslant j \leqslant k_{2, l}, 1 \leqslant l \leqslant m\right\}$. We define the memory in our SFDE by a collection of tame projections as follows

$$
\begin{gathered}
\Pi^{1}: C:=C\left([-\tau, 0), \mathbb{R}^{d}\right) \rightarrow \mathbb{R}^{d_{1}}, \quad \Pi^{2, l}: C \rightarrow \mathbb{R}^{d_{1, l}}, \\
\Pi^{1}(\eta):=\left(\eta\left(\tau_{1}^{1}\right), \eta\left(\tau_{1}^{2}\right), \ldots, \eta\left(\tau_{1}^{k_{1}}\right)\right), \quad \Pi^{2, l}(\eta):=\left(\eta\left(\tau_{2}^{1, l}\right), \eta\left(\tau_{2}^{2, l}\right), \ldots, \eta\left(\tau_{2}^{k_{2, l}, l}\right)\right)
\end{gathered}
$$

for all $\eta \in C$, and quasi-tame projections

$$
\Pi_{q}^{1}: C \rightarrow \mathbb{R}^{d_{1}^{q}}, \quad \Pi_{q}^{2, l}: C \rightarrow \mathbb{R}^{d_{2, l}^{q}}
$$

where $d_{1}=k_{1} d, d_{1}^{q}=k_{1} d, d_{2, l}=k_{2, l} d, d_{2, l}^{q}=k_{2, l} d$ are integer multiples of $d$, for $1 \leqslant l \leqslant m$. The quasi-tame projections have the following forms:

$$
\begin{aligned}
\Pi_{q}^{1}(\eta) & :=\left(\int_{-\tau}^{0} \sigma_{1}^{1}(\eta(s)) \mu_{1}^{1}(s) d s, \int_{-\tau}^{0} \sigma_{2}^{1}(\eta(s)) \mu_{2}^{1}(s) d s, \ldots, \int_{-\tau}^{0} \sigma_{k_{1}}^{1}(\eta(s)) \mu_{k_{1}}^{1}(s) d s\right) \\
\Pi_{q}^{2, l}(\eta) & :=\left(\int_{-\tau}^{0} \sigma_{1}^{2}(\eta(s)) \mu_{1}^{2}(s) d s, \int_{-\tau}^{0} \sigma_{2}^{2}(\eta(s)) \mu_{2}^{2}(s) d s, \ldots, \int_{-\tau}^{0} \sigma_{k_{2, l}}^{1}(\eta(s)) \mu_{k_{2, l}}^{2}(s) d s\right)
\end{aligned}
$$

for all $\eta \in C$. The functions $\sigma_{i}^{1}, \sigma_{j}^{2}, \mu_{i}^{1}, \mu_{j}^{2}$ are smooth. 
Consider the SFDE

$$
x(t)=\eta(0)+\int_{0}^{t} f\left(u, \Pi^{1}\left(x_{u}\right), \Pi_{q}^{1}\left(x_{u}\right)\right) d u+\sum_{l=1}^{m} \int_{0}^{t} g_{l}\left(u, \Pi^{2, l}\left(x_{u}\right), \Pi_{q}^{2, l}\left(x_{u}\right)\right) d W_{l}(u),
$$

for $\sigma \leqslant t \leqslant T$, with initial path

$$
x_{\sigma}=\eta \in H^{1, \infty}\left([-\tau, 0], \mathbb{R}^{d}\right) .
$$

Let $\pi:=\left\{-\tau=t_{-M}<t_{-M+1}<\ldots<t_{-1}<t_{0}=0<t_{1}<t_{2}<\ldots<t_{N-1}<t_{N}=T\right\}$ be a partition of $[-\tau, T]$, with equal size denoted by $|\pi|:=(T+\tau) /(N+M)$. The Euler approximations $y$ of the solution $x$ of (28) satisfy the following SFDE:

$$
\begin{aligned}
y(t)= & \eta(0)+\int_{0}^{t} f\left([u], \Pi^{1}\left(y_{[u]}\right), \Pi_{q}^{1}\left(y_{[u]}\right)\right) d u \\
& +\sum_{l=1}^{m} \int_{0}^{t} g_{l}\left([u], \Pi^{2, l}\left(y_{[u]}\right), \Pi_{q}^{2, l}\left(y_{[u]}\right)\right) d W_{l}(u),
\end{aligned}
$$

for $\sigma \leqslant t \leqslant T$, with initial path

$$
y_{\sigma}=\eta \in H^{1, \infty}\left([-\tau, 0], \mathbb{R}^{d}\right)
$$

Now we give the extension of Theorem 3.2. First we should adopt the following assumptions. (A.III) The functions

$$
f: \mathbb{R}^{+} \times \mathbb{R}^{d_{1}} \times \mathbb{R}^{d_{1}^{q}} \rightarrow \mathbb{R}^{d}, \quad g_{l}: \mathbb{R}^{+} \times \mathbb{R}^{d_{2, l}} \times \mathbb{R}^{d_{2, l}^{q}} \rightarrow \mathbb{R}^{d}, \quad 1 \leqslant l \leqslant m,
$$

are $C^{\infty}$ functions, whose derivatives of any order are bounded (but it is not assumed to be bounded itself).

(A.IV) For all $t \in[0, T], x(t ; 0, \eta)$ is non-degenerate, that is, the inverse Malliavin covariance matrix $\Gamma_{x(t ; 0, \eta)}$ which is defined in Definition 2.1 satisfies

$$
\left\|\Gamma_{x(t ; 0, \eta)}\right\| \in \mathbb{L}^{\infty-} .
$$

Theorem 5.1. Let $x(\cdot ; \sigma, \eta)$ be the unique solution of (28) with initial path $\eta \in$ $H^{1, \infty}\left([-\tau, 0], \mathbb{R}^{d}\right)$. Suppose that the coefficients $f, g$ satisfy the assumption (A.III) and $x(t ; 0, \eta)$ satisfies the assumption (A.IV). Let $\pi$ be a partition of $[-\tau, T]$ with equal size $|\pi|$, and $\eta^{\pi} \in H^{1, \infty}\left([-\tau, 0], \mathbb{R}^{d}\right)$ be the piecewise-linear approximation of $\eta$ along the partition $\pi$. Denote by $y(\cdot ; \sigma, \eta)$ the Euler approximation to $x(\cdot ; \sigma, \eta)$ associated with the partition $\pi$ and defined by (29). Then for bounded measurable function $\phi: \mathbb{R}^{d} \rightarrow \mathbb{R}$, there exists a positive constant $K$ and a positive integer $q$ such that

$$
\left|E \phi(x(t ; \sigma, \eta))-E \phi\left(y\left(t ; \sigma, \eta^{\pi}\right)\right)\right| \leqslant K\left(1+\|\eta\|_{1, \infty}^{q}\right)|\pi|
$$

for all $t \in[\sigma-\tau, T], \sigma \in[0, T]$ and all $\eta \in H^{1, \infty}\left([-\tau, 0], \mathbb{R}^{d}\right)$. The constant $K$ may depend on $T, q$ and the test function $\phi$, but is independent of $\pi, \eta, t \in[\sigma, T]$ and $\sigma \in[0, T]$.

The proof of Theorem 5.1 is essentially the same as the proof of Theorem 3.2, only now we should extend the local argument to the higher dimension. More precisely, we want to define a smooth functional $\psi\left(r_{t_{i} t_{n}}^{\pi}\right)$ such that outside a set where all of the Malliavin derivatives $D^{k}\left(\psi\left(r_{t_{i} t_{n}}^{\pi}\right)\right)$ vanish, we have a uniform control of the determinant of the Malliavin covariance matrix $\Sigma_{\lambda y\left(t_{n} ; t_{i}, x_{t_{i}}(\cdot ; 0, \eta)\right)+(1-\lambda) y\left(t_{n} ; t_{i-1}, x_{t_{i-1}}(\cdot ; 0, \eta)\right)}, 0 \leqslant \lambda \leqslant 1$. The last part of this section is just to sate this argument.

Let $\psi:[0, \infty) \mapsto \mathbb{R}$ be a $C_{b}^{\infty}$ function ( $\psi$ and all of its partial derivatives are bounded) such that

$$
1_{\left[0, \frac{1}{8}\right]} \leqslant \psi \leqslant 1_{\left[0, \frac{1}{4}\right]} .
$$


Let us introduce

$$
\begin{aligned}
r_{t_{i} t_{n}}^{\pi}= & \frac{\left|D\left(x\left(t_{n} ; 0, \eta\right)-\frac{1}{2} y\left(t_{n} ; t_{i}, x_{t_{i}}(\cdot ; 0, \eta)\right)-\frac{1}{2} y\left(t_{n} ; t_{i-1}, x_{t_{i-1}}(\cdot ; 0, \eta)\right)\right)\right|^{2}\left(1+\left\|\Sigma_{x\left(t_{n} ; 0, \eta\right)}\right\|_{2}^{2}\right)^{(d-1) / 2}}{\operatorname{det}\left(\Sigma_{x\left(t_{n} ; 0, \eta\right)}\right)} \\
& \vee \frac{\left|D\left(y\left(t_{n} ; t_{i}, x_{t_{i}}(\cdot ; 0, \eta)\right)-y\left(t_{n} ; t_{i-1}, x_{t_{i-1}}(\cdot ; 0, \eta)\right)\right)\right|^{2}\left(1+\left\|\Sigma_{x\left(t_{n} ; 0, \eta\right)}\right\|_{2}^{2}\right)^{(d-1) / 2}}{\operatorname{det}\left(\Sigma_{x\left(t_{n} ; 0, \eta\right)}\right)},
\end{aligned}
$$

where

$$
\left|D\left(x\left(t_{n} ; 0, \eta\right)\right)\right|^{2}=\sum_{j=1}^{d}\left|D\left(x_{j}\left(t_{n} ; 0, \eta\right)\right)\right|^{2} \quad \text { and } \quad\left\|\Sigma_{x\left(t_{n} ; 0, \eta\right)}\right\|_{2}
$$

is the Hilbert-Schmidt norm of the matrix $\Sigma_{x\left(t_{n} ; 0, \eta\right)}$. Observe that $\psi\left(r_{t_{i} t_{n}}^{\pi}\right) \in \mathbb{D}_{\infty}^{\infty-}$ and that

$$
\begin{aligned}
P\left(\psi\left(r_{t_{i} t_{n}}^{\pi}\right) \neq 1\right) & \leqslant P\left(\left|D\left(x\left(t_{n} ; 0, \eta\right)-\frac{1}{2} y\left(t_{n} ; t_{i}, x_{t_{i}}(\cdot ; 0, \eta)\right)-\frac{1}{2} y\left(t_{n} ; t_{i-1}, x_{t_{i-1}}(\cdot ; 0, \eta)\right)\right)\right|^{2}\right. \\
& \vee\left|D\left(y\left(t_{n} ; t_{i}, x_{t_{i}}(\cdot ; 0, \eta)\right)-y\left(t_{n} ; t_{i-1}, x_{t_{i-1}}(\cdot ; 0, \eta)\right)\right)\right|^{2} \\
& \left.\geqslant \frac{1}{8\left(1+\left\|\Sigma_{x\left(t_{n} ; 0, \eta\right)}\right\|_{2}^{2}\right)^{(d-1) / 2}}\right) .
\end{aligned}
$$

Applying the same procedure used in $\S 4$, we obtain that there exists a constant $C>0$ such that

$$
P\left(\psi\left(t_{t_{i} t_{n}}^{\pi}\right) \neq 1\right) \leqslant C|\pi|^{p},
$$

for all $p \in[2, \infty)$. Now we show that $\lambda y\left(t_{n} ; t_{i}, x_{t_{i}}(\cdot ; 0, \eta)\right)+(1-\lambda) y\left(t_{n} ; t_{i-1}, x_{t_{i-1}}(\cdot ; 0, \eta)\right)$ is non-degenerate on the set $\left\{\psi\left(r_{t_{i} t_{n}}^{\pi}\right) \neq 0\right\}$. First for any positive-definite $d$-dimensional matrix $A$, we have

$$
\|A\| \leqslant\|A\|_{2} \leqslant \sqrt{d}\|A\|
$$

and

$$
\lambda_{1}(A)^{d} \leqslant \operatorname{det}(A) \leqslant \lambda_{1}(A)\|A\|^{d-1},
$$

where $\|\cdot\|$ is operator norm, $\lambda_{1}(A)=\inf _{|\xi|=1} \xi^{\prime} A \xi$ and $|\xi|$ is the Euclidean norm of $\xi$ in $\mathbb{R}^{d}$. Thus, we have

$$
\frac{\operatorname{det}\left(\Sigma_{x\left(t_{n} ; 0, \eta\right)}\right)}{4\left(1+\left\|\Sigma_{x\left(t_{n} ; 0, \eta\right)}\right\|_{2}^{2}\right)^{(d-1) / 2}} \leqslant \frac{\lambda_{1}\left(\Sigma_{x\left(t_{n} ; 0, \eta\right)}\right)}{4} .
$$

Consequently, applying (32), we obtain

$$
\begin{aligned}
& \bigcup_{k=1}^{\infty}\left\{D^{k}\left(\psi\left(r_{t_{i} t_{n}}^{\pi}\right)\right) \neq 0\right\} \\
& \subset\left\{\left|D\left(x\left(t_{n} ; 0, \eta\right)-\frac{1}{2} y\left(t_{n} ; t_{i}, x_{t_{i}}(\cdot ; 0, \eta)\right)-\frac{1}{2} y\left(t_{n} ; t_{i-1}, x_{t_{i-1}}(\cdot ; 0, \eta)\right)\right)\right|^{2}\right. \\
& \vee\left|D\left(y\left(t_{n} ; t_{i}, x_{t_{i}}(\cdot ; 0, \eta)\right)-y\left(t_{n} ; t_{i-1}, x_{t_{i-1}}(\cdot ; 0, \eta)\right)\right)\right|^{2} \\
& \left.\leqslant \frac{\operatorname{det}\left(\Sigma_{x\left(t_{n} ; 0, \eta\right)}\right)}{4\left(1+\left\|\Sigma_{x\left(t_{n} ; 0, \eta\right)}\right\|_{2}^{2}\right)^{(d-1) / 2}}\right\} \\
& \subset\left\{\left|D\left(x\left(t_{n} ; 0, \eta\right)-\frac{1}{2} y\left(t_{n} ; t_{i}, x_{t_{i}}(\cdot ; 0, \eta)\right)-\frac{1}{2} y\left(t_{n} ; t_{i-1}, x_{t_{i-1}}(\cdot ; 0, \eta)\right)\right)\right|^{2}\right. \\
& \left.\vee\left|D\left(y\left(t_{n} ; t_{i}, x_{t_{i}}(\cdot ; 0, \eta)\right)-y\left(t_{n} ; t_{i-1}, x_{t_{i-1}}(\cdot ; 0, \eta)\right)\right)\right|^{2} \leqslant \frac{\lambda_{1}\left(\Sigma_{x\left(t_{n} ; 0, \eta\right)}\right)}{4}\right\} .
\end{aligned}
$$


Observe that for any $t \in[0,1]$, we have the following uniform lower bound

$$
\begin{aligned}
& \sqrt{\lambda_{1}\left(\Sigma_{\lambda y\left(t_{n} ; t_{i}, x_{t_{i}}(\cdot ; 0, \eta)\right)+(1-\lambda) y\left(t_{n} ; t_{i-1}, x_{t_{i-1}}(\cdot ; 0, \eta)\right)}\right)} \\
& =\inf _{|\xi|=1} \sqrt{\xi^{\prime} \Sigma_{\lambda y\left(t_{n} ; t_{i}, x_{t_{i}}(\cdot ; 0, \eta)\right)+(1-\lambda) y\left(t_{n} ; t_{i-1}, x_{t_{i-1}}(\cdot ; 0, \eta)\right)} \xi} \\
& =\inf _{|\xi|=1} \mid \sum_{i} \xi_{i} D\left(\frac{1}{2} y\left(t_{n} ; t_{i}, x_{t_{i}}(\cdot ; 0, \eta)\right)\right. \\
& \left.+\frac{1}{2} y\left(t_{n} ; t_{i-1}, x_{t_{i-1}}(\cdot ; 0, \eta)\right)+\left(\lambda-\frac{1}{2}\right)\left(y\left(t_{n} ; t_{i}, x_{t_{i}}(\cdot ; 0, \eta)\right)-y\left(t_{n} ; t_{i-1}, x_{t_{i-1}}(\cdot ; 0, \eta)\right)\right)\right) \mid \\
& \geqslant \inf _{|\xi|=1} \sqrt{\xi^{\prime} \sum_{\frac{1}{2} y\left(t_{n} ; t_{i}, x_{t_{i}}(\cdot ; 0, \eta)\right)+\frac{1}{2} y\left(t_{n} ; t_{i-1}, x_{t_{i-1}}(\cdot ; 0, \eta)\right)}} \\
& -\frac{1}{2} \sup _{|\xi|=1}\left|\sum_{i} \xi_{i} D\left(y^{i}\left(t_{n} ; t_{i}, x_{t_{i}}(\cdot ; 0, \eta)\right)-y^{i}\left(t_{n} ; t_{i-1}, x_{t_{i-1}}(\cdot ; 0, \eta)\right)\right)\right| \\
& \geqslant \sqrt{\lambda_{1}\left(\sum_{\frac{1}{2} y\left(t_{n} ; t_{i}, x_{t_{i}}(\cdot ; 0, \eta)\right)-\frac{1}{2} y\left(t_{n} ; t_{i-1}, x_{t_{i-1}}(\cdot ; 0, \eta)\right)}\right)} \\
& -\frac{1}{2}\left|D\left(y\left(t_{n} ; t_{i}, x_{t_{i}}(\cdot ; 0, \eta)\right)-y\left(t_{n} ; t_{i-1}, x_{t_{i-1}}(\cdot ; 0, \eta)\right)\right)\right| \\
& \geqslant \sqrt{\lambda_{1}\left(\Sigma_{x\left(t_{n} ; 0, \eta\right)}\right)}-\left|D\left(x\left(t_{n} ; 0, \eta\right)-\frac{1}{2} y\left(t_{n} ; t_{i}, x_{t_{i}}(\cdot ; 0, \eta)\right)-\frac{1}{2} y\left(t_{n} ; t_{i-1}, x_{t_{i-1}}(\cdot ; 0, \eta)\right)\right)\right| \\
& -\frac{1}{2}\left|D\left(y\left(t_{n} ; t_{i}, x_{t_{i}}(\cdot ; 0, \eta)\right)-y\left(t_{n} ; t_{i-1}, x_{t_{i-1}}(\cdot ; 0, \eta)\right)\right)\right| .
\end{aligned}
$$

Thus, if

$$
\begin{aligned}
& \left|D\left(x\left(t_{n} ; 0, \eta\right)-\frac{1}{2} y\left(t_{n} ; t_{i}, x_{t_{i}}(\cdot ; 0, \eta)\right)-\frac{1}{2} y\left(t_{n} ; t_{i-1}, x_{t_{i-1}}(\cdot ; 0, \eta)\right)\right)\right|^{2} \\
& \quad \vee\left|D\left(y\left(t_{n} ; t_{i}, x_{t_{i}}(\cdot ; 0, \eta)\right)-y\left(t_{n} ; t_{i-1}, x_{t_{i-1}}(\cdot ; 0, \eta)\right)\right)\right|^{2} \leqslant \lambda_{1}\left(\Sigma_{x\left(t_{n} ; 0, \eta\right)}\right) / 4,
\end{aligned}
$$

then we obtain

$$
\sqrt{\lambda_{1}\left(\Sigma_{\lambda y\left(t_{n} ; t_{i}, x_{t_{i}}(\cdot ; 0, \eta)\right)+(1-\lambda) y\left(t_{n} ; t_{i-1}, x_{t_{i-1}}(\cdot ; 0, \eta)\right)}\right)} \geqslant \frac{1}{4} \sqrt{\lambda_{1}\left(\Sigma_{x\left(t_{n} ; 0, \eta\right)}\right)}
$$

and, consequently, applying (31) and (32), we have

$$
\begin{aligned}
\operatorname{det}\left(\Sigma_{\lambda y\left(t_{n} ; t_{i}, x_{t_{i}}(\cdot ; 0, \eta)\right)+(1-\lambda) y\left(t_{n} ; t_{i-1}, x_{t_{i-1}}(\cdot ; 0, \eta)\right)}\right) & \geqslant \frac{1}{16^{d}} \lambda_{1}\left(\Sigma_{x\left(t_{n} ; 0, \eta\right)}\right)^{d} \\
& \geqslant \frac{\operatorname{det}\left(\Sigma_{x\left(t_{n} ; 0, \eta\right)}\right)^{d}}{16^{d}\left\|\Sigma_{x\left(t_{n} ; 0, \eta\right)}\right\|^{d(d-1)}} .
\end{aligned}
$$

Combining with (33) and (34), we obtain

$$
\begin{gathered}
\bigcup_{k=0}^{\infty}\left\{D^{k}\left(\psi\left(r_{t_{i} t_{n}}^{\pi}\right)\right) \neq 0\right\} \subset\left\{\operatorname{det}\left(\Sigma_{\lambda y\left(t_{n} ; t_{i}, x_{t_{i}}(; ; 0, \eta)\right)+(1-\lambda) y\left(t_{n} ; t_{i-1}, x_{t_{i-1}}(; ; 0, \eta)\right)}\right)\right. \\
\left.\geqslant \frac{\operatorname{det}\left(\Sigma_{x\left(t_{n} ; 0, \eta\right)}\right)^{d}}{16^{d}\left\|\Sigma_{x\left(t_{n} ; 0, \eta\right)}\right\|^{d(d-1)}}\right\} .
\end{gathered}
$$

Therefore, we obtain that the determinant of the inverse of the Malliavin covariance matrix $\Sigma_{\lambda y\left(t_{n} ; t_{i}, x_{t_{i}}(\cdot ; 0, \eta)\right)+(1-\lambda) y\left(t_{n} ; t_{i-1}, x_{t_{i-1}}(\cdot ; 0, \eta)\right)}$ is dominated by the random variable

$$
\frac{\operatorname{det}\left(\Sigma_{x\left(t_{n} ; 0, \eta\right)}\right)^{d}}{16^{d}\left\|\Sigma_{x\left(t_{n} ; 0, \eta\right)}\right\|^{d(d-1)}}
$$

on the set $\bigcup_{k=0}^{\infty}\left\{D^{k}\left(\psi\left(r_{t_{i} t_{n}}^{\pi}\right)\right) \neq 0\right\}$.

Acknowledgements. The authors are very grateful to Professor Jiagang Ren for valuable discussions. We would also like to thank the referee for their careful reading of this manuscript. 


\title{
References
}

1. E. Buckwar, R. Kuske, S.-E. A. Mohammed and T. Shardlow, 'Weak convergence of the Euler scheme for stochastic differential delay equations', LMS J. Comput. Math. 11 (2008) 60-69.

2. D. R. Bell and S. E. A. Mohammed, 'The Malliavin calculus and stohastic delay equations', J. Funct. Anal. 99 (1991) 75-99.

3. V. BAlly and D. TAlay, 'The law of the Euler scheme for stochastic differential equations: I. Convergence rate of the distribution function', Probab. Theory Related Fields 104 (1996) 43-60.

4. V. BAlly and D. TAlay, 'The law of the Euler scheme for stochastic differential equations: II. Convergence rate of the rate of the density', Monte Carlo Methods Appl. 2 (1996) 93-128.

5. N. Bouleau and F. Hirsch, Dirichlet forms and analysis on Wiener space, de Gruyter Studies in Mathematics (de Gruyter, Berlin, 1991).

6. E. Clément, A. Kohatsu-Higa and D. Lamberton, 'A duality approach for the weak approximation of stochastic differential equations', Ann. Appl. Probab. 16 (2006) 1124-1154.

7. Z. HUANG and J. YAN, Introduction to infinite dimensional stochastic analysis, Mathematics and its Applications 502 (Kluwer Academic Publishers, Dordrecht, 2000). Translated and revised from the 1997 Chinese edition (Science Press, Beijing).

8. P. E. Kloteden and E. Platen, Numerical solutions of stochastic differential equations (Springer, New York, 1995).

9. A. Kohatsu-Higa, 'Weak approximations, a Malliavin calculus approach', Math. Comp. 70 (2001) $135-172$.

10. S. Kuosuoka and D. Stroock, 'Applications of the Malliavin calculus, Part I', Stochastic analysis (Kata/Kyoto, 1982), North-Holland Math. Library 32 (North-Holland, Amsterdam, 1984) 271-306.

11. P. Maldiavin, Stochastic analysis, Grundlehren der Mathematischen Wissenschaften 313 (Springer, Berlin, 1997).

12. S. E. A. Moнамmed, Stochastic functional differential equations, Research Notes in Mathematics 99 (Pitman, Boston, 1984).

13. S. E. A. Монамmed, 'Stochastic differential systems with memory: theory, examples and applications', Stochastic analysis, Progress in Probability 42 (eds L. Decreusefond, J. Gjerde, B. Øksendal and A. S. Ustunel; Birkhäuser, Basel, 1998) 1-77.

14. D. Nualart, 'The Malliavin calculus and related topics', Probability and its Applications, 2nd edn. (Springer, Berlin, 2006).

15. D. Nualart and E. Pardoux, 'Stochastic calculus with anticipating integrands', Probab. Theory Related Fields 78 (1998) 535-581.

16. K. He, J. Ren and H. Zhang, 'Localization of fractional Wiener functionals and applications', Preprint, 2013, arXiv:1304.4316.

\author{
Hua Zhang \\ School of Statistics \\ Jiangxi University of Finance and Economics \\ Nanchang, Jiangxi 330013, PR China \\ and
}

School of Mathematics and Computational Science

Sun Yat-Sen University

Guangzhou, Guangdong 510275, PR China

zh860801@163.com 